Supplement of Biogeosciences, 14, 1773-1792, 2017

http://www.biogeosciences.net/14/1773/2017/

doi:10.5194/bg-14-1773-2017-supplement

(C) Author(s) 2017. CC Attribution 3.0 License.

(c) (i)

Supplement of

\title{
Spatial variability of organic matter molecular composition and elemental geochemistry in surface sediments of a small boreal Swedish lake
}

Julie Tolu et al.

Correspondence to: Julie Tolu (julietolu@ hotmail.com)

The copyright of individual parts of the supplement might differ from the CC-BY 3.0 licence. 


\section{Supplementary information}

Table S1. Identified organic compounds by Py-GC-MS along with their formula, molecular mass (M), retention time (RT), calculated and reference retention index (Kovats retention index, $\mathrm{RI}^{\mathrm{a}}$ ), references for the theoretical mass spectra (REF) and how the individual organic compounds have been grouped for the statistical analyses

\begin{tabular}{|c|c|c|c|c|c|c|c|c|}
\hline \multirow{2}{*}{ Name } & \multirow{2}{*}{ Formula } & \multirow{2}{*}{ M } & \multirow{2}{*}{ RT } & \multirow{2}{*}{$\begin{array}{c}\mathrm{RI}_{\text {this }} \\
\text { study }\end{array}$} & \multicolumn{2}{|c|}{ reference $\mathrm{RI}^{\mathrm{b}}$} & \multirow{2}{*}{ REF } & \multirow{2}{*}{ Compounds groups } \\
\hline & & & & & $\mathrm{RI}_{\text {estimated }}$ & $\mathrm{RI}_{\text {experimental }}$ & & \\
\hline \multicolumn{9}{|l|}{ Carbohydrates } \\
\hline 3-furaldehyde & $\mathrm{C} 5 \mathrm{H} 4 \mathrm{O} 2$ & 96 & 189.5 & N.D. ${ }^{c}$ & & & NIST, [1] & \multirow{9}{*}{$\begin{array}{l}\text { (Alkyl)furans \& } \\
\text { furanones }\end{array}$} \\
\hline 2-furaldehyde & $\mathrm{C} 5 \mathrm{H} 4 \mathrm{O} 2$ & 96 & 204.1 & N.D. & & & NIST, [1] & \\
\hline 2-acetyl-furan & $\mathrm{C} 6 \mathrm{H} 6 \mathrm{O} 2$ & 110 & 267.2 & N.D. & & & NIST, [1] & \\
\hline Methyl-3-furaldehyde & C6H6O2 & 110 & 269.8 & N.D. & & & NIST, [1] & \\
\hline $2(5 \mathrm{H})$-furanone & $\mathrm{C} 4 \mathrm{H} 4 \mathrm{O} 2$ & 84 & 270.5 & N.D. & & & NIST, [1] & \\
\hline Methyl-2-furaldehyde & C6H6O2 & 110 & 276.9 & N.D. & & & NIST, [1] & \\
\hline Dihydro-methyl-furanone & $\mathrm{C} 5 \mathrm{H} 6 \mathrm{O}$ & 98 & 277.8 & N.D. & & & {$[1]$} & \\
\hline 5-methyl-2(5H)-Furanone & $\mathrm{C} 5 \mathrm{H} 6 \mathrm{O} 2$ & 98 & 292.5 & 907 & 868 & $914-917$ & NIST, [1] & \\
\hline Methyl-2-furaldehyde & $\mathrm{C} 6 \mathrm{H} 6 \mathrm{O} 2$ & 110 & 312.8 & 920 & 920 & $924-987$ & NIST, [1] & \\
\hline 2-Furancarboxylic acid, methyl ester & C6H6O3 & 126 & 422.7 & & 909 & & NIST, [1] & \multirow{3}{*}{$\begin{array}{c}\text { Hydroxyl or } \\
\text { carboxy-furans \& } \\
\text { furanones }\end{array}$} \\
\hline 2,5-Dimethyl-4-hydroxy-3(2H)-furanone & $\mathrm{C} 6 \mathrm{H} 8 \mathrm{O} 3$ & 128 & 424.6 & 1088 & 1022 & $1023-1097$ & NIST & \\
\hline 5-(hydroxymethyl)-2-Furaldehyde & C6H6O3 & 126 & 551.9 & 1236 & 1163 & $1176-1236$ & NIST, [1] & \\
\hline 5,6-dihydro-2H-Pyran-2-one & C5H6O2 & 98 & 278.7 & N.D. & & & NIST & \multirow{2}{*}{ Pyrans } \\
\hline 4-hydroxy-5,6-dihydro(2H)-pyran-2-one & $\mathrm{C} 5 \mathrm{H} 6 \mathrm{O} 3$ & 114 & 344.2 & 941 & N.R. ${ }^{d}$ & N.R. & {$[1]$} & \\
\hline Dianhydrorhamnose & $\mathrm{C}_{6} \mathrm{H}_{8} \mathrm{O}_{3}$ & 128 & 383.4 & 1041 & N.R. & N.R. & {$[2]$} & Dianhydrorhamnose \\
\hline Levoglucosenone & $\mathrm{C} 6 \mathrm{H} 6 \mathrm{O} 3$ & 126 & 450.1 & 1119 & N.R. & 1070 & NIST & Levoglucosenone \\
\hline Levosugars (Levogalactosan) & C6H10O5 & 162 & 535.7 & 1375 & N.R. & N.R. & [1] & \multirow{4}{*}{ Anhydrosugars } \\
\hline Ahydrohexose & Unknown & & 729.0 & 1450 & N.R. & N.R. & {$[1]$} & \\
\hline Levosugars(Levomannosan) & $\mathrm{C} 6 \mathrm{H} 10 \mathrm{O} 5$ & 162 & 761.5 & 1447 & N.R. & N.R. & {$[1]$} & \\
\hline Levosugars (Levoglucosan) & C6H10O5 & 162 & 771.4 & 1493 & 1404 & $1486-1491$ & [1] & \\
\hline \multicolumn{9}{|l|}{ Chitin derived compounds } \\
\hline Acetamide & $\mathrm{C} 2 \mathrm{H} 5 \mathrm{NO}$ & 59 & 166.3 & N.D. & & & NIST & \multirow{4}{*}{$\begin{array}{l}\text { Chitin derived } \\
\text { compounds }\end{array}$} \\
\hline 3-acetamido-furan & $\mathrm{C} 6 \mathrm{H} 7 \mathrm{NO} 2$ & 125 & 555.4 & 1239 & N.R. & N.R. & NIST & \\
\hline 3-acetamido-4-pyrone & Unknown & 153 & 656.4 & 1353 & N.R. & N.R. & [3] & \\
\hline Oxazoline & Unknown & & 801.9 & 1548 & N.R. & N.R. & [3] & \\
\hline N-compounds & & & & & & & & \\
\hline Pyridine & $\mathrm{C} 5 \mathrm{H} 5 \mathrm{~N}$ & 79 & 151.9 & N.D. & & & NIST & \\
\hline 2-methyl-pyridine & $\mathrm{C} 6 \mathrm{H} 7 \mathrm{~N}$ & 93 & 197.6 & N.D. & & & NIST & (Alkyl)pyridines \\
\hline 3/4-methyl-pyridine & C6H7N & 93 & 234.6 & N.D. & & & NIST & \\
\hline 2-Acetylpyridine & C7H7NO & 121 & 377.2 & 1034 & N.R. & N.R. & NIST & \\
\hline 3-Acetylpyridine & $\mathrm{C} 7 \mathrm{H} 7 \mathrm{NO}$ & 121 & 404.0 & 1065 & N.R. & N.R. & NIST & Pyridines_O \\
\hline 2-Methyl-5-acetoxypyridine & $\mathrm{C} 8 \mathrm{H} 9 \mathrm{NO} 2$ & 151 & 734.1 & 1457 & N.R. & N.R. & NIST & \\
\hline Pyrrole & C4H5N & 67 & 154.2 & N.D. & & & NIST & \\
\hline Methyl-pyrrole & $\mathrm{C} 5 \mathrm{H} 7 \mathrm{~N}$ & 81 & 216.2 & N.D. & & & NIST & (Alkyl)pyrroles \\
\hline 2-formyl-pyrrole & C5H5NO & 95 & 354.2 & 1008 & 988 & $1005-1030$ & NIST & \\
\hline 2-acetyl-pyrrole & C6H7NO & 109 & 402.0 & 1062 & 1035 & $1026-1064$ & NIST & Pyrroles_O \\
\hline 2-Formyl-1-methylpyrrole & C6H7NO & & 459.9 & 1129 & 1077 & $1092-1105$ & NIST & \\
\hline 2,5-pyrroledione & $\mathrm{C} 4 \mathrm{H} 3 \mathrm{NO} 2$ & 97 & 335.9 & 935 & N.R. & N.R. & NIST & Pyrroledione \& \\
\hline 2,5-pyrrolidinedione & $\mathrm{C} 4 \mathrm{H} 5 \mathrm{NO} 2$ & 99 & 462.1 & 1131 & 934 & N.R. & NIST & pyrrolidinedione \\
\hline Benzeneacetonitrile & $\mathrm{C} 8 \mathrm{H} 7 \mathrm{~N}$ & 117 & 474.2 & 1147 & 1138 & $1089-1143$ & NIST & \\
\hline Benzenepropanenitrile & C9H9N & 131 & 563.9 & 1248 & 1238 & $1186-1242$ & NIST & Aromatic N \\
\hline Indole & $\mathrm{C} 8 \mathrm{H} 7 \mathrm{~N}$ & 117 & 609.9 & 1293 & 1174 & $1260-1303$ & NIST & \\
\hline Methyl-indole & $\mathrm{C} 9 \mathrm{H} 9 \mathrm{~N}$ & 131 & 686.1 & 1394 & 1288 & $1380-1410$ & NIST & Indoles \\
\hline Diketodipyrrole & Unknown & 186 & 922.7 & 1722 & N.R. & N.R. & [4] & Diketodipyrrole \\
\hline Diketopiperazine Pro-Ala & $\mathrm{C} 8 \mathrm{H} 12 \mathrm{~N} 2 \mathrm{O} 2$ & 168 & 938.0 & 1746 & N.R. & N.R. & {$[5,6]$} & \\
\hline Diketopiperazine Pro-Val & $\mathrm{C} 10 \mathrm{H} 16 \mathrm{~N} 2 \mathrm{O} 2$ & 196 & 989.4 & 1826 & N.R. & N.R. & {$[5,6]$} & \\
\hline Diketopiperazine Pro-Val & $\mathrm{C} 10 \mathrm{H} 16 \mathrm{~N} 2 \mathrm{O} 2$ & 196 & 1005.9 & 1852 & N.R. & N.R. & & \\
\hline Diketopiperazine Cyclo-Leu-Pro & $\mathrm{C} 11 \mathrm{H} 18 \mathrm{~N} 2 \mathrm{O} 2$ & 210 & 1049.0 & 1922 & N.R. & N.R. & NIST & Diketopıperazınes \\
\hline Diketopiperazine Pro-Pro & $\mathrm{C} 10 \mathrm{H} 14 \mathrm{~N} 2 \mathrm{O} 2$ & 194 & 1067.0 & 1953 & N.R. & N.R. & {$[5,6]$} & \\
\hline Diketopiperazine Pro-Phe & $\mathrm{C} 14 \mathrm{H} 16 \mathrm{~N} 2 \mathrm{O} 2$ & 244 & 1284.3 & 2352 & N.R. & N.R. & {$[5,6]$} & \\
\hline Alkylamide1 & Unknown & & 986.8 & 1821 & N.R. & N.R. & NIST & \\
\hline Alkylamide2 & Unknown & & 1081.1 & 1977 & N.R. & N.R. & NIST & \\
\hline Alkylamide3 & Unknown & & 1197.6 & 2185 & N.R. & N.R. & NIST & \\
\hline Alkylamide4 & Unknown & & 1237.3 & 2260 & N.R. & N.R. & NIST & \\
\hline Alkylamide 5 & Unknown & & 1304.2 & 2392 & N.R. & N.R. & NIST & Alkylamides \\
\hline Alkylamide6 & Unknown & & 1579.6 & 3020 & N.R. & N.R. & NIST & \\
\hline
\end{tabular}




\begin{tabular}{|c|c|c|c|c|c|c|c|c|}
\hline$n$-alkenes & & & & & & & & \\
\hline n-C9:1 & C9H18 & 126 & & & & & NIST & \multirow{4}{*}{ C9-16:1 } \\
\hline n-C13:1 & $\mathrm{C} 13 \mathrm{H} 26$ & 182 & 603.4 & 1286 & 1204 & $1187-1289$ & NIST & \\
\hline $\mathrm{n}-\mathrm{C} 14: 1$ & $\mathrm{C} 14 \mathrm{H} 28$ & 196 & 684.6 & 1392 & 1421 & $1389-1396$ & NIST & \\
\hline $\mathrm{n}-\mathrm{C} 16: 1$ & $\mathrm{C} 16 \mathrm{H} 32$ & 224 & 834.4 & 1593 & 1602 & $1590-1593$ & NIST & \\
\hline n-C17:1 & C17H34 & 238 & 903.6 & 1693 & N.R. & $1692-1703$ & NIST & \multirow{6}{*}{ C17-22:1 } \\
\hline n-C18:1 & С18H36 & 252 & 969.2 & 1794 & 1801 & $1788-1793$ & NIST & \\
\hline n-C19:1 & С19H38 & 266 & 1031.8 & 1894 & 1900 & $1883-1899$ & NIST & \\
\hline$n-C 20: 1$ & $\mathrm{C} 2 \mathrm{OH} 40$ & 280 & 1091.5 & 1994 & N.R. & 1889-1994 & NIST & \\
\hline $\mathrm{n}-\mathrm{C} 21: 1$ & $\mathrm{C} 21 \mathrm{H} 42$ & 294 & 1148.5 & 2096 & 2117 & 2060 & NIST & \\
\hline $\mathrm{n}-\mathrm{C} 22: 1$ & $\mathrm{C} 22 \mathrm{H} 44$ & 308 & 1203.2 & 2195 & 2198 & $2192-2195$ & NIST & \\
\hline$n-C 23: 1$ & $\mathrm{C} 23 \mathrm{H} 46$ & 322 & 1255.5 & 2295 & N.R. & $2288-2295$ & NIST & \multirow{4}{*}{ C23-26:1 } \\
\hline $\mathrm{n}-\mathrm{C} 24: 1$ & $\mathrm{C} 24 \mathrm{H} 48$ & 336 & 1305.7 & 2395 & N.R. & $2394-2396$ & NIST & \\
\hline$n-C 25: 1$ & $\mathrm{C} 25 \mathrm{H} 50$ & 350 & 1352.9 & 2498 & N.R. & $2483-2496$ & NIST & \\
\hline n-C26:1 & $\mathrm{C} 26 \mathrm{H} 52$ & 364 & 1400.5 & 2596 & N.R. & $2593-2596$ & NIST & \\
\hline$n-C 27: 1$ & C27H54 & 350 & 1445.6 & 2697 & N.R. & $2688-2694$ & NIST & \multirow{2}{*}{ C27-28:1 } \\
\hline n-C28:1 & C28H56 & 364 & 1488.5 & 2796 & N.R. & 2794-2797 & NIST & \\
\hline \multicolumn{9}{|l|}{ n-alkanes } \\
\hline $\mathrm{n}-\mathrm{C} 10: 0$ & $\mathrm{C} 10 \mathrm{H} 22$ & 142 & 347.4 & & & & NIST & \multirow{7}{*}{ C10-16:0 } \\
\hline $\mathrm{n}-\mathrm{C} 11: 0$ & $\mathrm{C} 11 \mathrm{H} 24$ & 156 & 435.0 & & & & NIST & \\
\hline $\mathrm{n}-\mathrm{C} 12: 0$ & $\mathrm{C} 12 \mathrm{H} 26$ & 170 & 515.5 & & & & NIST & \\
\hline$n-C 13: 0$ & C13H28 & 184 & 617.3 & & & & NIST & \\
\hline$n-C 14: 0$ & C14H30 & 198 & 690.7 & & & & NIST & \\
\hline$n-C 15: 0$ & $\mathrm{C} 15 \mathrm{H} 32$ & 212 & 767.1 & & & & NIST & \\
\hline $\mathrm{n}-\mathrm{C} 16: 0$ & $\mathrm{C} 16 \mathrm{H} 34$ & 226 & 839.5 & & & & NIST & \\
\hline $\mathrm{n}-\mathrm{C} 17: 0$ & $\mathrm{C} 17 \mathrm{H} 36$ & 240 & 908.1 & & & & NIST & \multirow{6}{*}{ C17-22:0 } \\
\hline$n-C 18: 0$ & C18H38 & 254 & 973.5 & & & & NIST & \\
\hline$n-C 19: 0$ & $\mathrm{C} 19 \mathrm{H} 40$ & 268 & 1035.7 & & & & NIST & \\
\hline n-C20:0 & $\mathrm{C} 2 \mathrm{OH} 42$ & 282 & 1095.0 & & & & NIST & \\
\hline $\mathrm{n}-\mathrm{C} 21: 0$ & $\mathrm{C} 21 \mathrm{H} 44$ & 296 & 1150.7 & & & & NIST & \\
\hline $\mathrm{n}-\mathrm{C} 22: 0$ & $\mathrm{C} 22 \mathrm{H} 46$ & 310 & 1206.1 & & & & NIST & \\
\hline n-C23:0 & $\mathrm{C} 23 \mathrm{H} 48$ & 324 & 1258.1 & & & & NIST & \multirow{4}{*}{$\mathrm{C} 23-26: 0$} \\
\hline $\mathrm{n}-\mathrm{C} 24: 0$ & $\mathrm{C} 24 \mathrm{H} 50$ & 338 & 1308.1 & & & & NIST & \\
\hline$n-C 25: 0$ & $\mathrm{C} 25 \mathrm{H} 52$ & 352 & 1355.1 & & & & NIST & \\
\hline $\mathrm{n}-\mathrm{C} 26: 0$ & $\mathrm{C} 26 \mathrm{H} 54$ & 366 & 1402.4 & & & & NIST & \\
\hline$n-C 27: 0$ & C27H56 & 380 & 1447.1 & & & & NIST & \multirow{8}{*}{$\mathrm{C} 27-35: 0$} \\
\hline$n-C 28: 0$ & C28H58 & 394 & 1490.2 & & & & NIST & \\
\hline $\mathrm{n}-\mathrm{C} 29: 0$ & $\mathrm{C} 29 \mathrm{H} 60$ & 408 & 1530.3 & & & & NIST & \\
\hline n-C30:0 & $\mathrm{C} 30 \mathrm{H} 62$ & 422 & 1571.8 & & & & NIST & \\
\hline $\mathrm{n}-\mathrm{C} 31: 0$ & C31H64 & 436 & 1610.9 & & & & NIST & \\
\hline $\mathrm{n}-\mathrm{C} 32: 0$ & С32H66 & 450 & 1648.6 & & & & NIST & \\
\hline$n-C 33: 0$ & C33H68 & 464 & 1685.0 & & & & NIST & \\
\hline$n-C 35: 0$ & $\mathrm{C} 35 \mathrm{H} 72$ & 492 & 1723.9 & & & & [7] & \\
\hline \multicolumn{9}{|l|}{ Alkan-2-ones } \\
\hline $2-\mathrm{K} \mathrm{C} 13$ & $\mathrm{C} 13 \mathrm{H} 26 \mathrm{O}$ & 198 & 765.4 & 1498 & 1449 & 1476-1498 & NIST & \multirow{3}{*}{ 2K C13-17 } \\
\hline $2-\mathrm{K} \mathrm{C} 16$ & $\mathrm{C} 16 \mathrm{H} 32 \mathrm{O}$ & 240 & 975.0 & 1803 & 1748 & $1780-1805$ & NIST & \\
\hline 2-K C17 & $\mathrm{C} 17 \mathrm{H} 34 \mathrm{O}$ & 254 & 1038.2 & 1904 & 1847 & $1875-1890$ & NIST & \\
\hline $2-\mathrm{K} \mathrm{C} 19$ & $\mathrm{C} 19 \mathrm{H} 38 \mathrm{O}$ & 282 & 1155.9 & 2109 & 2046 & $2087-2106$ & NIST & \\
\hline 2-K C20 & $\mathrm{C} 20 \mathrm{H} 40 \mathrm{O}$ & 396 & 1210.9 & 2209 & 2206 & N.R. & NIST & 2K C19-22 \\
\hline $2-\mathrm{K} \mathrm{C} 21$ & $\mathrm{C} 21 \mathrm{H} 42 \mathrm{O}$ & 310 & 1263.7 & 2311 & 2309 & N.R. & NIST & \\
\hline $2-\mathrm{K} \mathrm{C} 23$ & $\mathrm{C} 23 \mathrm{H} 46 \mathrm{O}$ & 338 & 1363.0 & 2517 & 2513 & N.R. & NIST & \\
\hline $2-\mathrm{K} \mathrm{C} 24$ & $\mathrm{C} 24 \mathrm{H} 48 \mathrm{O}$ & 352 & 1410.0 & 2617 & N.R. & N.R. & NIST & \\
\hline $2-\mathrm{K} \mathrm{C} 25$ & $\mathrm{C} 25 \mathrm{H} 50 \mathrm{O}$ & 366 & 1455.1 & 2719 & N.R. & N.R. & NIST & \\
\hline $2-\mathrm{K} \mathrm{C} 26$ & $\mathrm{C} 26 \mathrm{H} 52 \mathrm{O}$ & 380 & 1498.6 & 2821 & N.R. & N.R. & NIST & 2K C23-31 \\
\hline 2-K C27 & $\mathrm{C} 27 \mathrm{H} 54 \mathrm{O}$ & 394 & 1540.6 & 2925 & N.R. & N.R. & [7] & \\
\hline $2-\mathrm{K} \mathrm{C} 28$ & $\mathrm{C} 28 \mathrm{H} 56 \mathrm{O}$ & 408 & 1581.3 & 3024 & N.R. & N.R. & [7] & \\
\hline 2-K C29 & $\mathrm{C} 29 \mathrm{H} 58 \mathrm{O}$ & 422 & 1620.7 & 3126 & N.R. & N.R. & [7] & \\
\hline 2-K C31 & $\mathrm{C} 31 \mathrm{H} 62 \mathrm{O}$ & 450 & 1696.0 & 3328 & N.R. & N.R. & [7] & \\
\hline Phenols & & & & & & & & \\
\hline Phenol & C6H6O & 94 & 329.2 & 931 & 901 & $952-1004$ & NIST & \\
\hline 2- methyl-phenol & $\mathrm{C} 7 \mathrm{H} 8 \mathrm{O}$ & 108 & 396.2 & 1056 & 1014 & $1029-1039$ & NIST & \\
\hline 3/4- methyl-phenol & $\mathrm{C} 7 \mathrm{H} 8 \mathrm{O}$ & 108 & 414.9 & 1077 & 1014 & $1051-1063$ & NIST & \\
\hline Dimethyl-phenol & $\mathrm{C} 7 \mathrm{H} 8 \mathrm{O}$ & 122 & 418.4 & 1156 & 1127 & $1077-1130$ & NIST & Phenols \\
\hline Ethyl- phenol & $\mathrm{C} 8 \mathrm{H} 70 \mathrm{O}$ & 122 & 497.3 & 1177 & 1114 & $1106-1162$ & NIST & \\
\hline Propenyl-phenol & $\mathrm{C} 9 \mathrm{H} 10 \mathrm{O}$ & 134 & 646.7 & 1340 & 1203 & $1232-1258$ & NIST & \\
\hline Lignins & & & & & & & & \\
\hline Guaiacol (G) & $\mathrm{C} 7 \mathrm{H} 8 \mathrm{O} 2$ & 124 & 428.7 & 1093 & 1090 & $1052-1090$ & NIST & \\
\hline Ethyl-guaiacol (guaiacyl-2C) & $\mathrm{C} 9 \mathrm{H} 12 \mathrm{O} 2$ & 152 & 596.2 & 1279 & 1303 & $1243-1287$ & NIST & acols \\
\hline
\end{tabular}




\begin{tabular}{|c|c|c|c|c|c|c|c|c|}
\hline 4-vinyl-guaiacol (guaiacyl -2C) & $\mathrm{C} 9 \mathrm{H} 10 \mathrm{O} 2$ & 150 & 625.6 & 1311 & 1293 & $1272-1295$ & NIST & \\
\hline 4-propenyl-guaiacol (guaiacyl -3C) & $\mathrm{C} 10 \mathrm{H} 12 \mathrm{O} 2$ & 164 & 660.9 & 1359 & 1392 & $1339-1452$ & NIST & \\
\hline Vanillin (guaiacyl -1C or -aldehyde) & $\mathrm{C} 8 \mathrm{H} 8 \mathrm{O} 3$ & 152 & 695.9 & 1407 & 1392 & $1350-1447$ & NIST & \\
\hline 4-alleneguaiacol (guaiacyl -3C) & $\mathrm{C} 10 \mathrm{H} 10 \mathrm{O} 2$ & 162 & 741.0 & 1466 & N.R. & N.R. & {$[8]$} & \\
\hline $\begin{array}{l}\text { Acetovanillone (guaiacyl -2Cor } \\
\text { aldehyde) }\end{array}$ & $\mathrm{C} 9 \mathrm{H} 10 \mathrm{O} 3$ & 166 & 763.1 & 1495 & 1439 & $1447-1503$ & NIST & \\
\hline $\begin{array}{l}\text { Vanillic acid, methyl ester (guaiacyl -1C } \\
\text { or -acid) }\end{array}$ & $\mathrm{C} 9 \mathrm{H} 10 \mathrm{O} 4$ & 182 & 785.5 & 1525 & 1470 & $1463-1525$ & NIST & \\
\hline $\begin{array}{l}\text { Guaiacylacetone (guaiacyl -3Cor - } \\
\text { aldehyde) }\end{array}$ & $\mathrm{C} 10 \mathrm{H} 12 \mathrm{O} 3$ & 180 & 796.0 & 1540 & 1538 & $1488-1531$ & NIST & \\
\hline Syringol (syringyl) & $\mathrm{C} 8 \mathrm{H} 10 \mathrm{O} 3$ & 154 & 655.4 & 1352 & 1279 & 1349-1367 & NIST & \multirow{5}{*}{ Syringols } \\
\hline 4-vinyl-syringol (syringy-2C) & $\mathrm{C} 11 \mathrm{H} 14 \mathrm{O} 4$ & 210 & 819.5 & 1572 & N.R. & $1517-1573$ & NIST & \\
\hline 4-formyl-syringol (syringy-1C) & $\mathrm{C} 9 \mathrm{H} 10 \mathrm{O} 4$ & 182 & 887.6 & 1670 & 1581 & $1617-1670$ & NIST & \\
\hline 4-allenesyringol (syringy-3C) & $\mathrm{C} 11 \mathrm{H} 12 \mathrm{O} 3$ & 192 & 901.0 & 1690 & N.R. & N.R. & [8] & \\
\hline Acetosyringone (syringy-2C) & $\mathrm{C} 10 \mathrm{H} 12 \mathrm{O} 4$ & 196 & 961.1 & 1781 & 1628 & $1740-1744$ & NIST & \\
\hline \multicolumn{9}{|l|}{ Chlorophylls } \\
\hline Prist-1-ene & C19H38 & 266 & 929.0 & 1732 & N.R. & N.R. & {$[9]$} & \multirow{2}{*}{ Pristenes } \\
\hline Prist-2-ene & C19H38 & 266 & 936.3 & 1743 & N.R. & N.R. & [9] & \\
\hline Phytadiene 1 & $\mathrm{C} 20 \mathrm{H} 38$ & 278 & 999.1 & 1841 & N.R. & N.R. & [9] & \multirow{2}{*}{ Phytadienes } \\
\hline Phytadiene 2 & $\mathrm{C} 20 \mathrm{H} 38$ & 278 & 1026.0 & 1884 & N.R. & N.R. & [9] & \\
\hline \multicolumn{9}{|l|}{ Steroids } \\
\hline Cholest-2-ene & $\mathrm{C}_{27} \mathrm{H}_{46}$ & 370 & 1522.1 & 2880 & 2380 & N.R. & NIST & \multirow{6}{*}{ Steroids } \\
\hline Cholesta-3,5-diene & $\mathrm{C}_{27} \mathrm{H}_{44}$ & 368 & 1543.4 & 2932 & N.R. & N.R. & NIST & \\
\hline Stigmasta-5,22-dien-3-ol, acetate & $\mathrm{C}_{31} \mathrm{H}_{50} \mathrm{O}_{2}$ & 454 & 1601.1 & 3075 & 2879 & N.R. & NIST & \\
\hline Sitosterol & $\mathrm{C}_{29} \mathrm{H}_{50} \mathrm{O}$ & 414 & 1623.4 & 3133 & 2731 & $3173-3220$ & NIST & \\
\hline Cholesta-3,5-dien-7-one & $\mathrm{C}_{27} \mathrm{H}_{42} \mathrm{O}$ & 382 & 1668.7 & 3255 & 2562 & N.R. & NIST & \\
\hline Stigmasta-3,5-dien-7-one & $\mathrm{C} 28 \mathrm{H} 46 \mathrm{O}$ & 410 & 1750.1 & 3462 & 2696 & N.R. & NIST & \\
\hline \multicolumn{9}{|l|}{ Tocopherols } \\
\hline$\gamma$-Tocopherol & $\mathrm{C} 28 \mathrm{H} 48 \mathrm{O} 2$ & 416 & 1594.1 & 3057 & 3036 & 3055 & NIST & \multirow{2}{*}{ Tocopherols } \\
\hline$\alpha$-Tocopherol & $\mathrm{C} 29 \mathrm{H} 50 \mathrm{O} 2$ & 430 & 1632.2 & 3157 & 3149 & 3111 & NIST & \\
\hline \multicolumn{9}{|l|}{ Hopanoids } \\
\hline Trinosphopane & unknown & & 1526.4 & 2890 & N.R. & N.R. & {$[10]$} & \multirow{6}{*}{ Hopanoids } \\
\hline Norhopene (triterpene C29) & unknown & & 1548.9 & 2945 & N.R. & N.R. & [10] & \\
\hline 22,29,30-trisnorhop-17(21)-ene & $\mathrm{C}_{27} \mathrm{H}_{44}$ & 368 & 1553.8 & 2957 & N.R. & N.R. & [11] & \\
\hline 22,29,30-trisnorhop-16(17)-ene & $\mathrm{C}_{27} \mathrm{H}_{44}$ & 368 & 1567.1 & 2989 & N.R. & N.R. & [11] & \\
\hline Norhopane (C30/C31?) & unknown & & 1630.7 & 3153 & N.R. & N.R. & [10] & \\
\hline 25-norhopene (C30/C31?) & unknown & & 1659.0 & 3229 & N.R. & N.R. & [10] & \\
\hline \multicolumn{9}{|l|}{$($ Poly)aromatics } \\
\hline Benzene & $\mathrm{C}_{6} \mathrm{H}_{6}$ & 78 & 113.9 & N.D. & & & NIST & Benzene \\
\hline Benzaldehyde & $\mathrm{C}_{7} \mathrm{H}_{6} \mathrm{O}$ & 106 & 311.2 & 919 & 982 & $925-966$ & NIST & Benzaldehyde \\
\hline Acetyl-benzene & $\mathrm{C} 8 \mathrm{H} 8 \mathrm{O}$ & 120 & 408.1 & 1069 & 1029 & 1041-1078 & NIST & Acetyl-benzene \\
\hline Styrene & $\mathrm{C} 8 \mathrm{H} 8$ & 104 & 254.2 & N.D. & & & NIST & \multirow{5}{*}{ Benzenes C2-9 } \\
\hline Ethyl-methyl-benzene & $\mathrm{C} 9 \mathrm{H} 12$ & 120 & 368.2 & 1024 & N.R. & $945-973$ & NIST & \\
\hline Indene & $\mathrm{C} 9 \mathrm{H} 8$ & 120 & 389.7 & 1048 & 1014 & $1029-1051$ & NIST & \\
\hline Benzene C7 & $\mathrm{C} 13 \mathrm{H} 20$ & 176 & 664.9 & 1365 & 1390 & $1337-1350$ & NIST & \\
\hline Benzene C9 & $\mathrm{C} 15 \mathrm{H} 24$ & 204 & 822.1 & 1576 & 1555 & $1552-1586$ & NIST & \\
\hline 1,2-dihydro-naphthalene & $\mathrm{C} 10 \mathrm{H} 10$ & 130 & 484.9 & 1160 & 1149 & $1137-1166$ & NIST & \multirow{7}{*}{ (Poly)aromatics } \\
\hline 2,3-dihydro-inden-1-one & $\mathrm{C} 9 \mathrm{H} 8 \mathrm{O}$ & 132 & 599.8 & 1283 & 1218 & $1218-1320$ & NIST & \\
\hline 1/2-methyl-napthalene & $\mathrm{C} 11 \mathrm{H} 10$ & 142 & 612.1 & 1295 & 1345 & $1263-1298$ & NIST & \\
\hline 2Amethyl-napthalene & $\mathrm{C} 11 \mathrm{H} 10$ & 142 & 626.7 & 1313 & 1345 & $1267-1298$ & NIST & \\
\hline Biphenyl & $\mathrm{C} 12 \mathrm{H} 10$ & 154 & 680.8 & 1386 & 1367 & $1338-1392$ & NIST & \\
\hline Fluorene & C13H10 & 166 & 836.9 & 1596 & 1494 & $1549-1611$ & NIST & \\
\hline Anthracene & $\mathrm{C} 14 \mathrm{H} 10$ & 178 & 972.2 & 1798 & 1782 & $1740-1800$ & NIST & \\
\hline
\end{tabular}

${ }^{a}$ Kovats retention index (RI) is used to convert retention times into system-independent constants. The RI of a certain chemical compound is its retention time normalized to the retention times of adjacently eluting $n$-alkanes and is determined as follow:

$\mathrm{RI}=100 \mathrm{x}\left[\mathrm{n}+\frac{\log (\text { RT unknown })-\log (R T n)}{\log (R T N)-\log (R T n)}\right.$

With, $\mathrm{n}$ is the number of carbon in the adjacently eluting smaller $n$-alkane; RT unknown is the retention time of the compound to identify, $\mathrm{RT} \mathrm{n}$ is the retention time of the adjacently elution smaller $n$-alkane, and RT $\mathrm{N}$ is the retention time of the adjacently eluting larger $n$ alkane.

${ }^{\mathrm{b}}$ reference RI: the reference RI values were found on the website "NIST Chemistry Webbook" (http://webbook.nist.gov/chemistry/) and/or in the 'NIST/EPA/NIH 2011' library included in the software "NIST MS Search v.2.0"; the reference RI values reported in this table have been either assessed or determined experimentally for non-polar GC column and temperature gradient GC program.;

${ }^{c}$ N.D.: the RI value could not be determined in this study because the adjacently eluting $n$-alkanes needed to calculate the RI could not be identified.

${ }^{\mathrm{d}}$ N.R.: no reference RI values were found in the NIST Webbook or library. 


\section{References (the complete references are given in the manuscript):}

[1] Faix et al. (1991) Holz als Roh- und Werkstoff, 49: 213-219; [2] Schellenkens et al. (2009) Organic geochemistry 40: 678-691; [3] Gupta and Cody (2011), in N.S. Gupta (ed.), Chitin, Topics in Geobiology 34, Springer Science+Busines Media ; [4] Schellenkens et al. (2014) Organic Geochemistry 77: 32-42; [5] Chen et al., (2009) Journal of Food Science, 74: 100-105; [6] Fabbri et al., (2012) Journal of Analytical and Applied Pyrolysis, 95: 145-155; [7] For these long-chain $n$-alkanes and alkan-2-ones, the number of C in the molecule has been determined based on the highest $\mathrm{m} / \mathrm{z}$ present in the mass spectra that corresponds to the molecular mass and on the order of elution; [8] Faix et al. (1990) Holz als Roh- und Werkstoff, 48: 281-285; [9] Nguyen et al. (2005) Organic Geochemistry, 34: 483-497; [10] Gill (1997) Chapter 16. Analytical techniques in organic chemistry; in: Modern analytical Geochemistry; Taylor \& Francis, New York (USA), pp. 243-272; [11] Meredith et al. (2008) Organic Geochemistry, 39: 1243-1248 
Table S2. Sediment elemental geochemistry variables for the 44 studied sediment samples (to be continued)

\begin{tabular}{|c|c|c|c|c|c|c|c|c|c|c|c|}
\hline unit & $\begin{array}{c}\text { WD } \\
\mathrm{m}\end{array}$ & $\begin{array}{c}\mathrm{BD} \\
\mathrm{g} \mathrm{cm}^{-3}\end{array}$ & $\begin{array}{c}\mathrm{bSi} \\
\%\end{array}$ & $\begin{array}{c}\text { LOI } \\
\%\end{array}$ & $\begin{array}{c}{[\mathrm{S}]} \\
\mathrm{mg} \mathrm{kg}^{-1}\end{array}$ & $\begin{array}{c}{[\mathrm{Br}]} \\
\mathrm{mg} \mathrm{kg}^{-1}\end{array}$ & $\begin{array}{c}{[\mathrm{Cu}]} \\
\mathrm{mg} \mathrm{kg}^{-1}\end{array}$ & $\begin{array}{c}{[\mathrm{Ni}]} \\
\mathrm{mg} \mathrm{kg}^{-1}\end{array}$ & $\begin{array}{c}{[\mathrm{Hg}]} \\
\mu \mathrm{g} \mathrm{kg}^{-1}\end{array}$ & $\begin{array}{c}{[\mathrm{Pb}]} \\
\mathrm{mg} \mathrm{kg}^{-1}\end{array}$ & $\begin{array}{c}{[\mathrm{Zn}]} \\
\mathrm{mg} \mathrm{kg}^{-1}\end{array}$ \\
\hline N1 & 3.1 & 0.057 & 15 & 57 & 16620 & 134 & 29 & 21 & 283 & 151 & 285 \\
\hline $\mathrm{N} 2$ & 7.4 & 0.088 & 6 & 58 & 18050 & 127 & 39 & 23 & 510 & 168 & 320 \\
\hline N3 & $\underline{1.6}$ & 0.078 & 14 & 34 & 5570 & 216 & 34 & $\underline{10}$ & 261 & 170 & $\underline{43}$ \\
\hline N4 & $\overline{7.4}$ & 0.056 & 22 & 37 & 9700 & 138 & 33 & $\overline{17}$ & 288 & 120 & $\overline{121}$ \\
\hline N5 & 4 & 0.073 & 19 & 36 & 9740 & 174 & 26 & 18 & 187 & 114 & 195 \\
\hline N6 & 7.1 & 0.061 & 14 & 40 & 9770 & 176 & 27 & 18 & 291 & 208 & 199 \\
\hline N7 & 4 & 0.063 & 11 & 40 & 16240 & 168 & 34 & 23 & 309 & 236 & 332 \\
\hline N8 & 5.3 & 0.064 & 15 & 40 & 9960 & 138 & 28 & 17 & 224 & 175 & 218 \\
\hline N9 & 7.7 & 0.054 & 11 & 48 & 14570 & 214 & 34 & 20 & 288 & 187 & 248 \\
\hline N10 & 2.5 & 0.080 & 21 & 27 & 4990 & 129 & 37 & $\underline{10}$ & 290 & 261 & 50 \\
\hline N11 & 3.9 & 0.068 & 19 & 32 & 17000 & 134 & 36 & 21 & 288 & 226 & 254 \\
\hline E1 & 2 & 0.061 & 15 & 36 & 15440 & 119 & 28 & 19 & 235 & 208 & 188 \\
\hline E2 & 6.7 & 0.087 & 11 & 36 & 11510 & 129 & 26 & 19 & 242 & 118 & 195 \\
\hline E3 & 3.5 & 0.075 & 14 & 45 & 8240 & 177 & 19 & 16 & 198 & 109 & 213 \\
\hline E4 & 6 & 0.086 & 13 & 39 & 11600 & 158 & 27 & 19 & 235 & 152 & 228 \\
\hline E5 & 9.4 & 0.092 & 9 & 41 & 11260 & 145 & 29 & 19 & 265 & 138 & 200 \\
\hline E6 & 10 & 0.035 & 13 & 42 & 11350 & 150 & 30 & 20 & 309 & $\underline{422}$ & 222 \\
\hline M1 & 3 & 0.084 & $\underline{25}$ & 25 & 4840 & 146 & 33 & $\underline{10}$ & 264 & $\overline{235}$ & 49 \\
\hline M2 & 3.7 & 0.075 & $\overline{20}$ & 35 & 9640 & 167 & 22 & $\overline{20}$ & 193 & 120 & 239 \\
\hline M3 & 7.9 & 0.083 & 8 & 35 & 17560 & 120 & 36 & 22 & 326 & 206 & 279 \\
\hline M4 & 3.8 & 0.608 & 2 & 3 & 2960 & 11 & 7 & 9 & 34 & 33 & 61 \\
\hline M5 & 1.9 & $\underline{0.127}$ & 23 & 18 & 5000 & 120 & 13 & $\underline{10}$ & $\underline{117}$ & 135 & 74 \\
\hline M6 & 1.8 & $\overline{0.104}$ & 16 & $\underline{10}$ & $\underline{4685}$ & $\underline{71}$ & $\underline{12}$ & $\overline{18}$ & $\overline{141}$ & 175 & 80 \\
\hline S1 & 5.7 & 0.083 & 17 & $\overline{30}$ & $\overline{6640}$ & $\overline{104}$ & $\overline{27}$ & 19 & 181 & 103 & 144 \\
\hline $\mathrm{S} 2$ & 6.5 & 0.091 & 23 & 27 & 6600 & 101 & 25 & 14 & 177 & 97 & 111 \\
\hline S3 & 9.5 & 0.047 & 12 & 35 & 7410 & 157 & 31 & 14 & 263 & 145 & 104 \\
\hline S4 & 14 & 0.035 & 10 & 32 & 6295 & 133 & 29 & 13 & 242 & 180 & 173 \\
\hline S5 & 3.7 & 0.101 & 11 & 32 & 8940 & 91 & 15 & 12 & 177 & 105 & 146 \\
\hline S6 & 12.5 & 0.043 & 15 & 42 & 6040 & 172 & 31 & 18 & 196 & $\underline{58}$ & 104 \\
\hline S9 & 21 & 0.040 & 6 & 45 & 12650 & 175 & 44 & 23 & 507 & $\overline{244}$ & 299 \\
\hline S10 & 12.8 & 0.053 & 7 & 40 & 9230 & 187 & 49 & 21 & 370 & 188 & 196 \\
\hline S11 & 14.8 & 0.058 & 6 & 37 & 12460 & 159 & 41 & 21 & 428 & 256 & 308 \\
\hline S12 & 23.5 & 0.033 & $\underline{4}$ & 50 & 20650 & $\underline{225}$ & 56 & 26 & 740 & 279 & 380 \\
\hline S13 & 16.5 & 0.048 & $\overline{12}$ & 38 & 11780 & $\overline{160}$ & 45 & 22 & 491 & 258 & 303 \\
\hline S14 & 9.1 & 0.046 & 6 & 32 & 18260 & 119 & 43 & 20 & 385 & 279 & 336 \\
\hline S15 & 2.9 & 0.737 & 2 & 4 & 2180 & 21 & 11 & 15 & 21 & 10 & 39 \\
\hline S16 & 4.2 & 0.097 & 23 & 20 & 5420 & 90 & 24 & 18 & 162 & 120 & 100 \\
\hline S17 & 18.2 & 0.047 & $\underline{4}$ & 41 & 13980 & 170 & 48 & 25 & 461 & 295 & 404 \\
\hline S18 & 24.5 & 0.027 & $\overline{5}$ & 51 & $\underline{29190}$ & 212 & 64 & $\underline{27}$ & 858 & 277 & 425 \\
\hline S19 & 9.5 & 0.036 & 9 & 34 & $\overline{13790}$ & 153 & 39 & $\overline{18}$ & 368 & 224 & 199 \\
\hline S21 & 11 & 0.060 & 5 & 34 & 4950 & 153 & 28 & 15 & 187 & 112 & 118 \\
\hline S22 & 19.8 & 0.057 & 5 & 40 & 13950 & 164 & 48 & 24 & 533 & 299 & 415 \\
\hline S23 & 3.6 & 0.024 & 10 & 52 & 18370 & 123 & 39 & 20 & 547 & 243 & 255 \\
\hline S24 & $\underline{24.3}$ & $\underline{0.016}$ & $\underline{4}$ & 54 & 28840 & 175 & $\underline{75}$ & $\underline{27}$ & $\underline{1152}$ & 273 & $\underline{445}$ \\
\hline
\end{tabular}

The font colors of the sample ID cells correspond to the six clusters identified by the cluster analysis on the elemental geochemistry dataset, for which the sediments M4 and S15 (shown to be outliers) were discarded.

The bold and underlined numbers corresponds to the minimal and maximal value, and the numbers in bold correspond to the values that are below or above $20 \%$ of the maximal and minimal values respectively. 
Table S2. Continuation Sediment elemental geochemistry variables for the 44 studied sediment samples

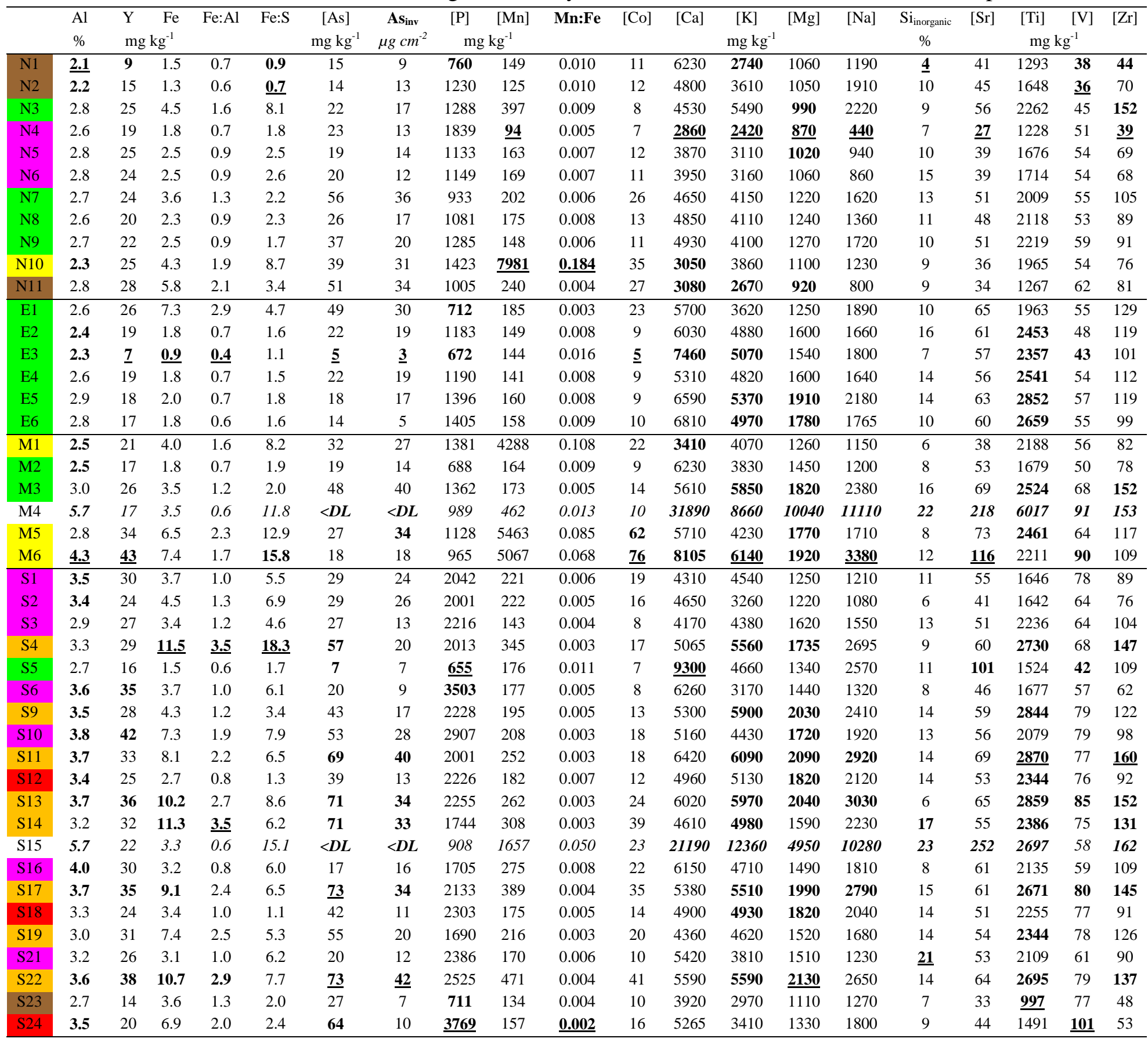

The font colors of the sample ID cells correspond to the six clusters identified by the cluster analysis on the elemental geochemistry dataset, for which the sediments M4 and S15 (shown to be outliers) were discarded.

The bold and underlined numbers corresponds to the minimal and maximal value, and the numbers in bold correspond to the values that are below or above $20 \%$ of the maximal and minimal values respectively. 
Table S3. Average of the sediment elemental geochemistry variables for the whole-lake, the six clusters and the two outliers

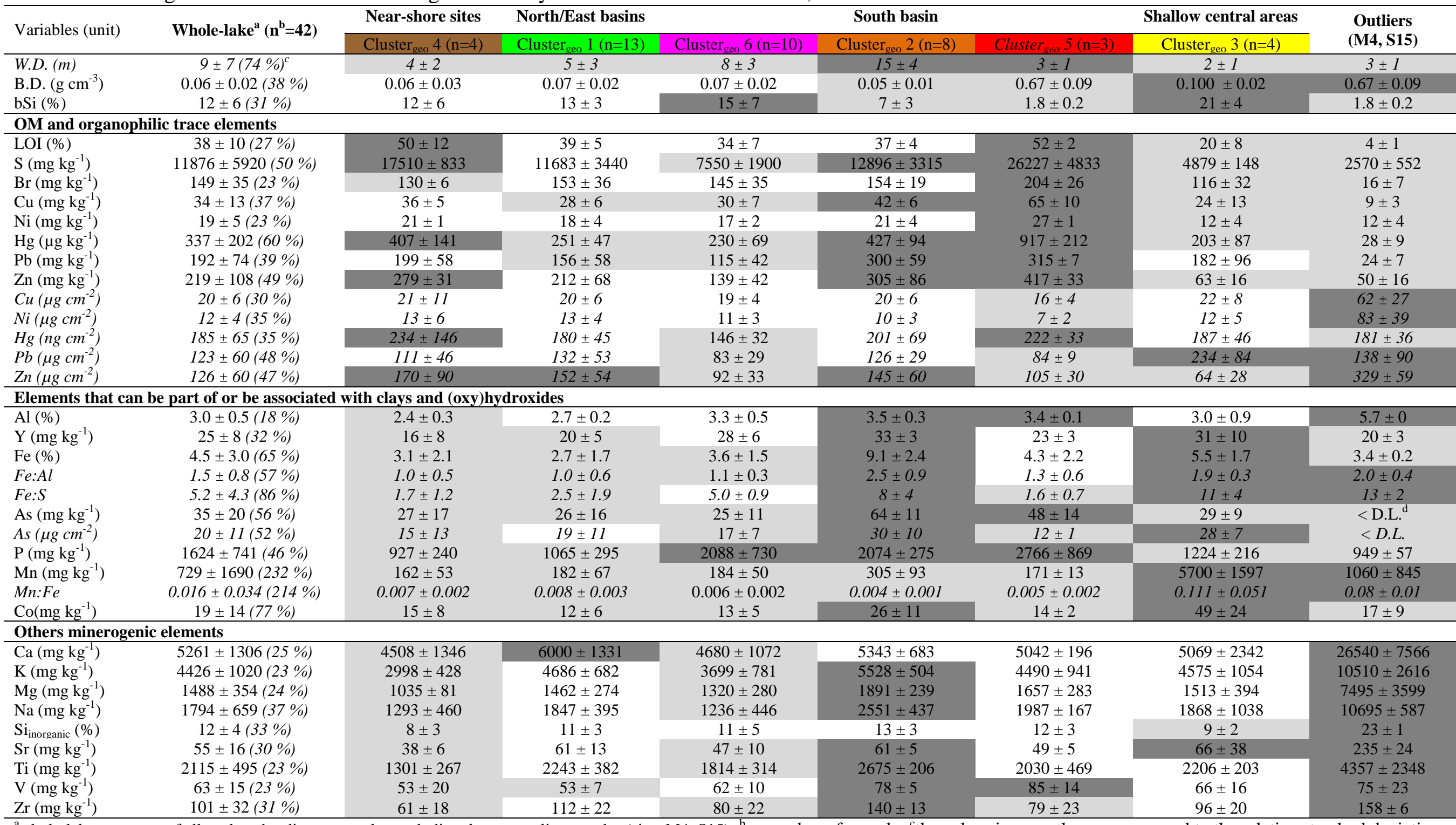

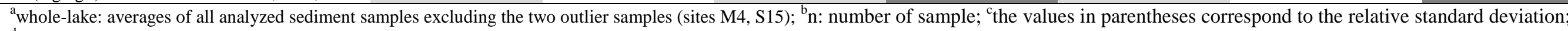
${ }^{\mathrm{d} D}$ D.L.: detection limit; The six clusters are presented in Fig. $1 \mathrm{~b}$ in the manuscript.

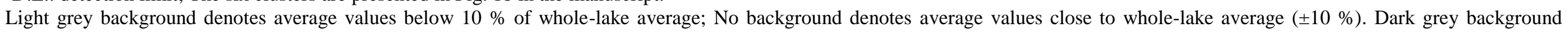
denotes average values above $10 \%$ of whole-lake average. The variables in italic are passive variables in the PCA and cluster analyses. 


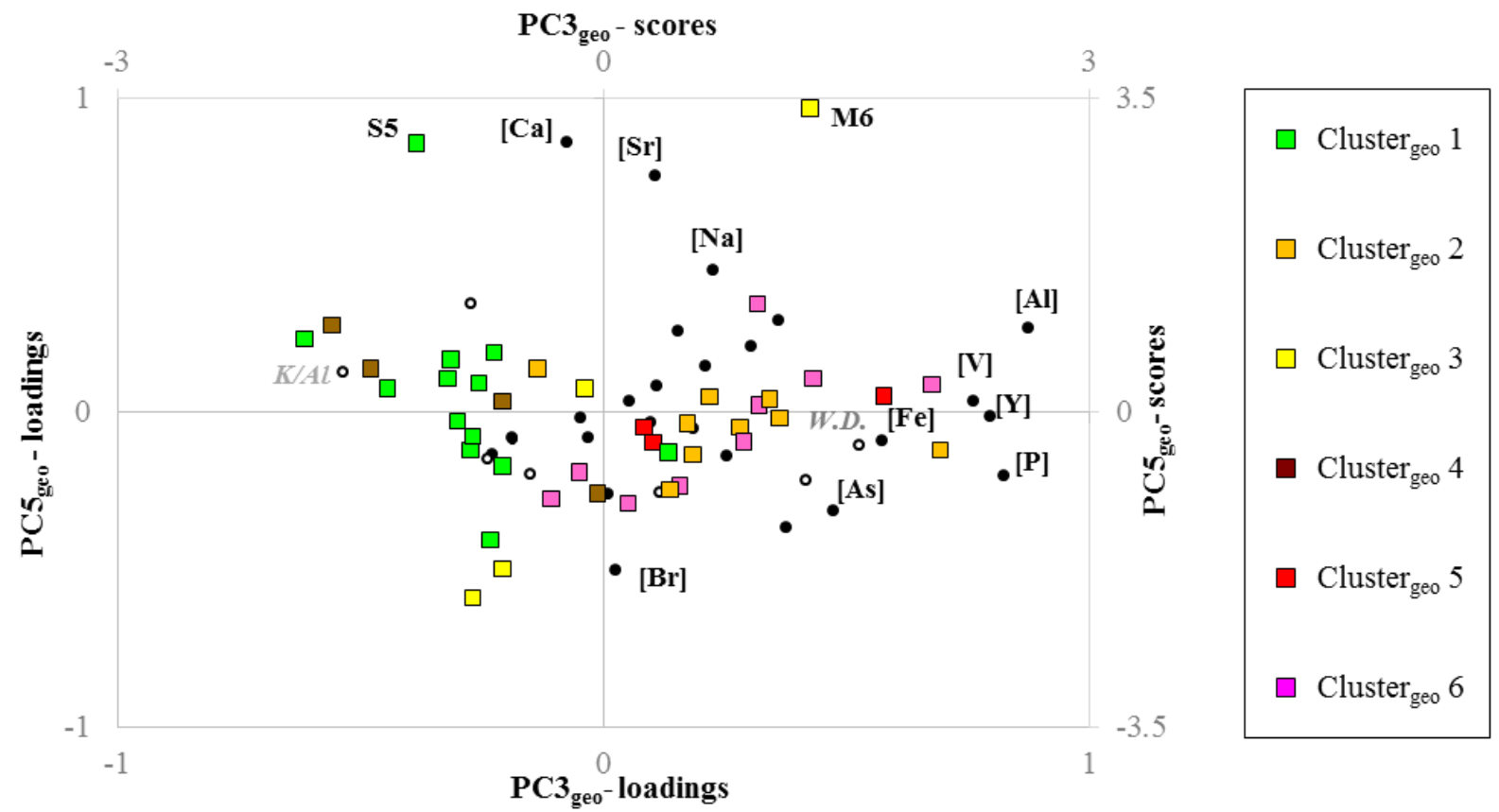

Fig. S1 Combined loading- and score-plots for PCs 3-5 of the elemental geochemistry dataset. For the PC-loadings, filled circles correspond to active variables, and others variables (empty circle and italics letter) were added passively. Sediment samples are colored according to the results of the cluster analysis.

This figure shows that $\mathrm{PC}_{\text {geo }}$ (10\% of total variance) separates $\mathrm{Ca}, \mathrm{Na}$ and $\mathrm{Sr}$ on the positive side from $\mathrm{Br}$ on the negative side. No reasonable interpretation could be made for this $\mathrm{PC} 5_{\text {geo }}$, which appears to be driven by only two samples which are split into two different clusters by the cluster analysis. 
Table S4. Sediment OM molecular composition variables for the 42 sediment samples analysed by Py-GC/MS (to be continued)

\begin{tabular}{|c|c|c|c|c|c|c|c|c|c|c|c|c|c|c|c|c|c|}
\hline & \multicolumn{6}{|c|}{ Carbohydrates } & \multirow[b]{2}{*}{$\begin{array}{l}\text { Chitin- } \\
\text { derived } \\
\text { compounds }\end{array}$} & \multicolumn{10}{|c|}{ N-compounds } \\
\hline & $\begin{array}{c}\text { (Alkyl)- } \\
\text { furans \& } \\
\text { furanones }\end{array}$ & $\begin{array}{l}\text { Hydroxy- or } \\
\text { carboxy-furans } \\
\text { \& furanones }\end{array}$ & Pyrans & $\begin{array}{l}\text { Dianhydro- } \\
\text { rhamnose }\end{array}$ & $\begin{array}{l}\text { Levoglu- } \\
\text { cosenone }\end{array}$ & $\begin{array}{l}\text { Anhydro } \\
\text {-sugars }\end{array}$ & & $\begin{array}{c}\text { (Alkyl)- } \\
\text { pyridines }\end{array}$ & Pyridines_O & $\begin{array}{l}\text { (Alkyl)- } \\
\text { pyrroles }\end{array}$ & Pyrroles_O & $\begin{array}{l}\text { Pyrroledione \& } \\
\text { pyrrolidinedione }\end{array}$ & $\underset{\mathbf{N}}{\text { Aromatic }}$ & Indoles & $\begin{array}{l}\text { Diketodi- } \\
\text { pyrrole }\end{array}$ & $\begin{array}{l}\text { Diketopi- } \\
\text { perazines }\end{array}$ & $\begin{array}{c}\text { Alkyl- } \\
\text { amides }\end{array}$ \\
\hline N1 & 10 & 5.1 & 4.5 & 2.0 & 2.2 & 3.1 & 2.9 & 0.42 & 0.68 & 2.1 & 0.61 & 1.20 & 0.40 & 1.40 & 1.10 & 1.90 & 0.46 \\
\hline N2 & $\underline{8}$ & 5.1 & 3.6 & 1.3 & 2.3 & 3.1 & 3.3 & 0.37 & 0.69 & 2.0 & 0.66 & 1.10 & 0.40 & 1.40 & $\underline{1.20}$ & 1.20 & 0.47 \\
\hline N3 & $\overline{11}$ & 5.9 & 1.7 & 0.8 & 1.5 & 2.5 & 1.2 & 0.11 & 0.62 & 1.8 & 0.86 & 0.60 & 0.60 & 1.20 & $\overline{0.50}$ & 1.30 & 0.74 \\
\hline N4 & 17 & 4.2 & 4.7 & 2.2 & 3.1 & 2.9 & 4.0 & 0.40 & $\underline{0.91}$ & 2.5 & 1.13 & 1.50 & 0.60 & 1.70 & 0.80 & 1.80 & 0.83 \\
\hline N5 & 13 & 4.0 & 3.7 & 1.7 & 2.2 & 6.4 & 2.9 & 0.34 & $\overline{0.65}$ & 2.2 & 1.00 & 1.40 & 0.70 & 1.40 & 0.70 & 1.50 & 0.61 \\
\hline N6 & 12 & 4.1 & 3.8 & 1.6 & 2.8 & 8.1 & 2.2 & 0.28 & 0.69 & 1.8 & 0.79 & 1.30 & 0.50 & 1.30 & 0.60 & 1.50 & 0.70 \\
\hline N7 & 13 & 4.0 & 3.8 & 1.8 & 2.3 & 4.3 & 2.7 & 0.31 & 0.71 & 2.0 & 0.81 & 1.20 & 0.60 & 1.40 & 0.70 & 1.50 & 0.73 \\
\hline N8 & 12 & 4.0 & 4.1 & 2.0 & 2.3 & 8.1 & 2.7 & 0.32 & 0.69 & 1.8 & 0.82 & 1.20 & 0.50 & 1.30 & 0.70 & 1.60 & 0.63 \\
\hline N9 & 13 & 4.6 & 4.3 & 1.6 & 2.6 & 2.3 & 2.5 & 0.30 & 0.65 & 1.9 & 0.75 & 1.10 & 0.50 & 1.30 & 0.80 & 1.60 & 0.74 \\
\hline N10 & 19 & 7.0 & 4.1 & 1.8 & 2.7 & 2.2 & 2.5 & 0.20 & 0.85 & 2.4 & 1.33 & 1.50 & 0.80 & 1.50 & 0.70 & 1.50 & 0.49 \\
\hline N11 & 16 & 3.2 & 2.6 & 1.5 & 2.2 & 2.2 & 1.9 & 0.20 & 0.73 & 2.4 & 0.96 & 1.20 & 0.80 & 1.50 & 0.70 & 1.30 & 0.69 \\
\hline E1 & 10 & 3.2 & 2.2 & 1.2 & 1.7 & 2.0 & 1.1 & 0.11 & 0.57 & 1.8 & 0.74 & 0.90 & 0.70 & 1.30 & 0.60 & 1.10 & 0.46 \\
\hline E2 & 10 & 4.2 & 3.8 & 1.8 & 2.3 & 8.1 & 2.6 & 0.27 & 0.70 & 1.9 & 0.75 & 1.10 & $\mathbf{0 . 4 0}$ & 1.30 & 0.60 & 1.50 & 0.73 \\
\hline E3 & 9 & 5.1 & 5.0 & 2.1 & 2.0 & 4.0 & 2.5 & 0.31 & 0.69 & 1.7 & 0.67 & 1.20 & $\underline{\mathbf{0 . 3 0}}$ & 1.30 & 0.80 & 1.30 & 0.56 \\
\hline E4 & 11 & 4.3 & 4.6 & 2.0 & 2.3 & 6.2 & 3.2 & 0.34 & 0.81 & $\overline{2.1}$ & 0.82 & 1.30 & $\overline{0.50}$ & 1.40 & 0.70 & 1.70 & 0.74 \\
\hline E5 & 10 & 4.0 & 4.0 & 1.9 & 2.3 & 5.6 & 3.2 & 0.30 & 0.70 & 2.0 & 0.79 & 1.40 & 0.50 & 1.40 & 0.70 & 1.80 & 0.80 \\
\hline E6 & 10 & 4.4 & 3.8 & 2.0 & 1.9 & 6.8 & 3.6 & 0.32 & 0.72 & 1.8 & 0.87 & 1.50 & 0.50 & 1.70 & 0.70 & 1.90 & 0.79 \\
\hline M1 & 18 & 7.5 & 3.2 & 1.3 & 2.6 & 2.2 & 1.4 & 0.20 & 0.68 & 2.6 & 1.19 & 1.30 & 0.80 & 1.40 & 0.40 & 1.20 & 0.31 \\
\hline M2 & 14 & 4.5 & 5.2 & $\underline{2.7}$ & 2.2 & 8.9 & 3.2 & 0.36 & 0.77 & 1.9 & 0.91 & 1.70 & 0.60 & 1.60 & 0.70 & 1.70 & 0.54 \\
\hline M3 & 11 & 3.3 & 2.6 & $\overline{1.2}$ & 2.0 & 6.2 & 2.6 & 0.29 & 0.71 & 2.1 & 0.83 & 1.10 & 0.60 & 1.50 & 0.80 & 1.60 & 0.49 \\
\hline M5 & 21 & $\underline{0.8}$ & 1.2 & $\underline{0.3}$ & 1.5 & 1.0 & $\underline{0.2}$ & $\underline{0.06}$ & 0.17 & 2.1 & $\underline{0.51}$ & $\underline{0.20}$ & 0.60 & $\underline{\mathbf{0 . 5 0}}$ & $\underline{0.40}$ & $\underline{\mathbf{0 . 3 0}}$ & 0.15 \\
\hline M6 & 24 & $\overline{1.6}$ & $\overline{1.7}$ & $\overline{0.6}$ & 2.3 & 1.1 & $\overline{0.3}$ & $\overline{\mathbf{0 . 0 7}}$ & 0.50 & 2.7 & $\overline{0.77}$ & $\overline{\mathbf{0 . 3 0}}$ & 1.10 & $\overline{0.90}$ & $\overline{1.00}$ & $\overline{1.00}$ & $\underline{0.06}$ \\
\hline S1 & 20 & 3.2 & 3.2 & 1.5 & 2.5 & 2.1 & 2.3 & 0.40 & 0.76 & 3.0 & 1.27 & 1.40 & 1.00 & 1.80 & 0.70 & 1.20 & $\overline{0.46}$ \\
\hline S2 & 22 & 3.2 & 2.5 & 1.7 & 2.1 & 1.6 & 1.7 & 0.33 & 0.74 & 3.2 & 1.27 & 1.00 & 1.10 & 1.90 & 0.60 & 0.90 & 0.24 \\
\hline S3 & 18 & 4.8 & 4.9 & 2.3 & 2.7 & $\underline{0.8}$ & 3.8 & 0.45 & 0.86 & 2.7 & 1.28 & 1.60 & 0.80 & 1.70 & 0.80 & 1.80 & 0.64 \\
\hline S4 & 17 & 3.1 & 2.2 & 1.3 & 1.7 & $\overline{1.8}$ & 1.6 & 0.36 & 0.76 & 3.4 & 1.16 & 0.80 & 1.30 & 1.80 & 0.70 & 0.90 & 0.27 \\
\hline S5 & 11 & 5.4 & 4.4 & 1.9 & 1.9 & $\underline{11.0}$ & 1.7 & 0.34 & 0.59 & 1.7 & 0.69 & 1.30 & 0.50 & 1.30 & 0.90 & 1.80 & 0.34 \\
\hline S6 & 15 & 3.6 & 2.9 & 1.7 & 1.8 & 2.5 & 4.0 & 0.17 & 0.85 & 2.7 & 1.33 & 1.40 & 0.80 & 1.80 & 0.80 & 1.40 & 0.65 \\
\hline S9 & 13 & 4.1 & 3.2 & 1.8 & 1.9 & 3.2 & 3.6 & 0.37 & 0.80 & 2.4 & 0.96 & 1.10 & 0.70 & 1.60 & 0.90 & 2.00 & 0.91 \\
\hline S10 & 16 & 3.7 & 2.5 & 1.5 & 2.0 & 2.7 & 3.4 & 0.40 & 0.89 & 3.4 & 1.37 & 1.10 & 0.90 & 1.70 & 0.90 & 1.40 & 0.58 \\
\hline S11 & 16 & 3.7 & 3.0 & 1.7 & 2.5 & 2.2 & 2.3 & 0.31 & 0.79 & 2.5 & 1.19 & 0.90 & 1.00 & 1.60 & 0.80 & 1.20 & 0.44 \\
\hline $\mathrm{S} 12$ & 12 & 3.7 & 3.2 & 1.5 & 2.2 & 1.7 & 4.2 & $\underline{0.46}$ & 0.75 & 2.8 & 0.90 & 1.50 & 0.80 & 1.90 & 1.00 & $\underline{2.60}$ & 1.17 \\
\hline S13 & 14 & 3.7 & 2.3 & 1.0 & 1.7 & 2.0 & $\overline{2.0}$ & $\overline{0.32}$ & 0.65 & 2.8 & 1.07 & 0.80 & 1.00 & 1.60 & 0.80 & $\overline{1.40}$ & 1.08 \\
\hline S14 & 20 & 3.6 & 3.0 & 1.5 & 2.6 & 2.1 & 1.7 & 0.37 & 0.79 & 3.4 & 1.10 & 0.90 & 1.30 & 1.70 & 0.80 & 1.30 & 0.33 \\
\hline S16 & $\underline{28}$ & 3.9 & 2.6 & 2.0 & $\underline{3.1}$ & 2.2 & 0.9 & 0.08 & 0.48 & $\underline{3.5}$ & 1.20 & $\underline{1.70}$ & 1.30 & 1.70 & 0.40 & 0.80 & 0.09 \\
\hline S17 & $\overline{14}$ & 3.8 & 2.8 & 1.5 & $\overline{1.7}$ & 2.2 & 2.6 & 0.30 & 0.81 & $\overline{2.6}$ & 1.07 & $\overline{0.80}$ & 1.00 & 1.60 & 0.80 & 1.50 & 0.47 \\
\hline S18 & 13 & 3.6 & 3.2 & 1.5 & 2.1 & 2.9 & 3.7 & 0.40 & 0.69 & 2.8 & 0.94 & 1.30 & 0.80 & 1.90 & 0.90 & $\underline{2.60}$ & 1.12 \\
\hline S19 & 21 & 3.8 & 3.5 & 1.6 & 2.7 & 0.9 & 2.5 & 0.32 & 0.85 & 2.9 & 1.27 & 1.30 & 1.00 & 1.60 & 0.70 & 1.30 & 0.39 \\
\hline S21 & 18 & 3.6 & 4.3 & 2.3 & 2.1 & 2.6 & 3.4 & 0.37 & 0.85 & 2.5 & $\underline{1.37}$ & 1.50 & 0.90 & 1.50 & 0.70 & 1.40 & 0.49 \\
\hline S22 & 14 & 3.3 & 2.0 & 1.3 & 1.7 & 1.8 & 2.1 & 0.36 & 0.72 & 2.7 & $\overline{1.09}$ & 0.70 & 1.00 & 1.60 & 0.90 & 1.20 & 0.38 \\
\hline S23 & 17 & 6.0 & $\underline{5.3}$ & 1.6 & 2.9 & 8.2 & 1.3 & 0.35 & 0.57 & 1.9 & 0.55 & 1.20 & 0.70 & 1.50 & 0.90 & 1.40 & 0.56 \\
\hline S24 & 11 & 3.6 & 2.7 & 1.8 & 1.3 & 1.9 & 3.4 & $\mathbf{0 . 3 7}$ & 0.86 & 3.1 & 0.85 & 1.60 & 1.40 & $\underline{\mathbf{3 . 1 0}}$ & 0.90 & 2.30 & 1.66 \\
\hline
\end{tabular}

The font colors of the sample ID cells correspond to the six clusters identified by the cluster analysis on the OM molecular composition dataset.

The bold and underlined numbers corresponds to the minimal and maximal value, and the numbers in bold correspond to the values that are below or above $20 \%$ of the maximal and minimal values respectively. 
Table S4. Continuation Sediment OM molecular composition variables for the 42 sediment samples analysed by Py-GC/MS (to be continued)

\begin{tabular}{|c|c|c|c|c|c|c|c|c|c|c|c|c|c|c|c|c|}
\hline & \multicolumn{3}{|c|}{ Lignins } & \multicolumn{2}{|c|}{ Chlorophylls } & \multicolumn{4}{|c|}{$n$-alkenes } & \multicolumn{4}{|c|}{$n$-alkanes } & \multicolumn{3}{|c|}{ Alkan-2-ones } \\
\hline & Phenols & Syringols & Guaiacols & Pristenes & Phytadienes & \begin{tabular}{|c|} 
C9-16:1 \\
\end{tabular} & C17-22:1 & C23-26:1 & C27-28:1 & \begin{tabular}{|l|} 
C13-16:0 \\
\end{tabular} & C17-22:0 & C23-26:0 & C27-35:0 & 2K C13-17 & $2 \mathrm{~K} \mathrm{C19-22}$ & 2K C23-31 \\
\hline N1 & 10.6 & 1.9 & 10.6 & 3.7 & 1.6 & 2.0 & 5.2 & 3.9 & 1.2 & 1.7 & 3.1 & 1.9 & 2.3 & $\underline{0.6}$ & 0.24 & 1.7 \\
\hline N2 & $\underline{11.4}$ & $\overline{1.4}$ & 7.7 & 3.0 & 1.7 & 2.3 & 5.8 & 3.0 & 0.9 & 1.9 & 3.2 & 3.2 & 6.3 & $\overline{\mathbf{0 . 6}}$ & 0.21 & 1.5 \\
\hline N3 & 9.0 & 0.6 & 3.6 & 2.8 & 1.3 & 3.8 & 7.1 & 4.8 & 1.3 & 2.7 & 4.5 & 4.1 & 6.1 & $\frac{0}{1.0}$ & 0.40 & 3.2 \\
\hline N4 & 7.6 & 0.3 & 2.3 & 2.3 & 3.5 & 2.8 & 5.1 & 2.1 & 0.6 & 1.9 & 3.4 & 2.4 & 4.1 & 1.4 & 0.24 & 1.0 \\
\hline N5 & 7.3 & 0.4 & 3.0 & 3.2 & 1.7 & 3.5 & 6.7 & 3.0 & 1.0 & 2.6 & 4.2 & 2.7 & 3.9 & 1.3 & 0.35 & 1.5 \\
\hline N6 & 7.3 & 0.4 & 3.8 & 3.2 & 1.9 & 2.8 & 6.2 & 3.4 & 0.8 & 1.9 & 3.5 & 3.1 & 6.1 & 1.1 & 0.28 & 1.9 \\
\hline N7 & 7.6 & 0.5 & 3.8 & 3.2 & 1.9 & 3.1 & 6.5 & 4.1 & 1.2 & 2.1 & 4.0 & 2.8 & 4.3 & 1.1 & 0.34 & 2.6 \\
\hline N8 & 7.2 & 0.4 & 3.6 & 3.2 & 1.6 & 3.1 & 6.6 & 3.4 & 1.1 & 2.3 & 3.9 & 3.0 & 4.9 & 1.1 & 0.33 & 2.0 \\
\hline N9 & 8.7 & 0.6 & 4.1 & 3.7 & 2.0 & 2.9 & 6.6 & 4.0 & 1.2 & 2.3 & 4.0 & 2.8 & 4.3 & 0.8 & 0.36 & 2.5 \\
\hline N10 & 9.5 & 0.2 & 1.8 & 1.5 & 1.5 & 3.0 & 5.2 & 2.1 & 0.4 & 2.4 & 3.1 & 2.7 & 3.5 & 1.0 & 0.19 & 0.6 \\
\hline N11 & 6.6 & 0.2 & 2.1 & 2.5 & 2.3 & 3.9 & 6.7 & 3.5 & 1.4 & 2.6 & 4.4 & 4.8 & 6.6 & 1.4 & 0.30 & 1.0 \\
\hline E1 & 8.1 & 0.3 & 3.5 & $\underline{4.6}$ & 1.4 & 4.0 & $\underline{8.9}$ & $\underline{5.4}$ & $\overline{1.3}$ & 2.7 & 5.1 & 3.9 & 5.6 & 1.0 & 0.35 & $\underline{\mathbf{3 . 3}}$ \\
\hline E2 & 7.4 & 0.5 & 3.7 & $\overline{3.4}$ & 1.5 & 3.1 & $\overline{7.1}$ & 3.6 & 1.2 & 2.2 & 4.1 & 3.4 & 5.4 & 0.9 & 0.30 & 2.3 \\
\hline E3 & 8.8 & 1.4 & 7.5 & 3.2 & 1.5 & 2.5 & 6.1 & 3.8 & 1.0 & 1.7 & 3.5 & 3.2 & 6.2 & 0.6 & 0.31 & 2.3 \\
\hline $\mathrm{E} 4$ & 7.3 & 0.5 & 3.7 & 3.2 & 1.9 & 2.9 & 6.6 & 3.6 & 1.0 & 2.0 & 3.9 & 2.6 & 4.8 & 1.1 & 0.31 & 2.3 \\
\hline E5 & 7.9 & 0.6 & 4.1 & 3.5 & 1.5 & 3.0 & 6.6 & 3.8 & 1.0 & 2.3 & 4.1 & 2.8 & 4.7 & 1.0 & 0.36 & 2.3 \\
\hline E6 & 8.0 & 0.7 & 4.6 & 3.1 & 1.8 & 2.9 & 6.2 & 3.6 & 1.1 & 2.4 & 3.8 & 2.4 & 4.3 & 1.0 & 0.40 & 2.1 \\
\hline M1 & 8.0 & 0.2 & 1.9 & 2.1 & 1.3 & 3.8 & 6.2 & 3.0 & 0.5 & 2.6 & 3.5 & 2.7 & 3.6 & 0.9 & 0.15 & 0.8 \\
\hline M2 & 7.0 & 0.7 & 4.0 & 2.2 & 1.9 & 2.8 & 5.4 & 2.6 & 0.8 & 2.1 & 3.3 & 2.5 & 3.5 & 1.1 & 0.27 & 1.2 \\
\hline M3 & 6.9 & 0.3 & 3.3 & 2.3 & 2.0 & 3.1 & 5.6 & 2.6 & 0.6 & 2.2 & 3.5 & 4.5 & 10.1 & 1.2 & 0.23 & 1.3 \\
\hline M5 & 4.4 & 0.1 & 1.1 & 0.4 & 0.2 & 4.1 & 3.8 & 0.6 & 0.1 & 2.3 & 1.6 & 8.8 & 21.3 & 1.6 & 0.02 & $\underline{0.1}$ \\
\hline M6 & $\overline{7.7}$ & $\overline{0.2}$ & $\overline{1.5}$ & $\overline{0.6}$ & $\overline{1.0}$ & 3.8 & 4.0 & $\overline{1.5}$ & 0.1 & 2.4 & $\overline{2.1}$ & $\overline{6.7}$ & $\overline{11.8}$ & 1.6 & $\overline{\mathbf{0 . 0 3}}$ & $\overline{0.3}$ \\
\hline $\mathrm{S} 1$ & 8.0 & 0.3 & 2.4 & 2.5 & 1.8 & 4.6 & 6.4 & 2.2 & 0.3 & 3.2 & 4.6 & 2.2 & 1.9 & 1.8 & 0.34 & 0.4 \\
\hline $\mathrm{S} 2$ & 6.9 & 0.2 & 2.1 & 2.7 & 2.2 & $\underline{5.1}$ & 6.9 & 2.1 & 0.3 & 3.7 & 4.6 & 2.0 & 1.4 & 1.9 & 0.31 & 0.3 \\
\hline $\mathrm{S} 3$ & 7.8 & 0.4 & 2.8 & 2.6 & 2.1 & $\frac{3.7}{3.7}$ & 5.8 & 2.2 & 0.7 & 2.8 & 4.1 & 1.5 & 1.6 & 1.6 & 0.44 & 0.7 \\
\hline S4 & 9.1 & 0.3 & 2.9 & 3.4 & 1.5 & 4.8 & 6.7 & 2.5 & 0.4 & 4.1 & 4.7 & 1.8 & 1.8 & 1.9 & 0.38 & 0.8 \\
\hline S5 & 9.6 & 1.8 & $\underline{13.5}$ & 2.2 & 1.2 & 2.1 & 4.4 & 2.6 & 0.7 & $\overline{1.7}$ & 2.5 & $\underline{1.4}$ & 1.4 & 0.7 & 0.15 & 0.7 \\
\hline S6 & 7.9 & 0.4 & 2.8 & 2.9 & 1.9 & 4.6 & 6.9 & 2.5 & 0.5 & 3.7 & 5.4 & 1.8 & 1.7 & 1.8 & 0.53 & 0.9 \\
\hline S9 & 8.4 & 0.4 & 2.9 & 2.8 & 1.9 & 3.7 & 6.3 & 3.1 & 1.0 & 2.7 & 4.6 & 2.5 & 3.5 & 1.5 & 0.44 & 1.6 \\
\hline S10 & 8.0 & 0.3 & 2.3 & 2.6 & 2.1 & 4.4 & 6.6 & 2.1 & 0.6 & 3.2 & 4.6 & 1.6 & 1.7 & 2.0 & 0.47 & 0.7 \\
\hline S11 & 8.8 & 0.3 & 2.9 & 3.2 & 1.6 & 4.3 & 6.8 & 2.6 & 0.7 & 2.9 & 4.5 & 1.9 & 2.3 & 1.8 & 0.40 & 0.9 \\
\hline $\mathrm{S} 12$ & 8.6 & 0.4 & 2.5 & 2.6 & 2.8 & 3.4 & 5.8 & 2.7 & 1.0 & 2.6 & 4.1 & 2.2 & 3.6 & 1.6 & 0.34 & 1.2 \\
\hline S13 & 9.0 & 0.3 & 2.6 & 2.8 & 1.4 & 4.5 & 6.6 & 2.3 & 0.6 & 3.1 & 4.2 & 2.9 & 3.9 & 2.0 & 0.82 & 1.1 \\
\hline S14 & 8.5 & 0.2 & 2.2 & 2.9 & 3.3 & 4.0 & 5.6 & 2.2 & 0.4 & 2.3 & 3.2 & 1.6 & 2.4 & 1.7 & $\overline{0.28}$ & 0.6 \\
\hline S16 & 6.4 & 0.2 & 2.1 & 2.0 & 1.3 & 4.5 & 5.8 & 1.5 & $\underline{0.1}$ & 3.0 & 3.4 & 1.7 & $\underline{1.1}$ & 1.4 & 0.05 & 0.1 \\
\hline S17 & 9.0 & 0.3 & 2.7 & 3.0 & 2.0 & 4.1 & 6.6 & 3.2 & 0.7 & 2.8 & 4.6 & 2.4 & 3.0 & 1.8 & 0.44 & 1.1 \\
\hline S18 & 8.7 & 1.2 & 3.8 & 2.4 & 2.9 & 3.1 & 5.1 & 2.6 & 0.9 & 2.4 & 3.7 & 2.2 & 3.3 & 1.4 & 0.30 & 1.0 \\
\hline S19 & 8.7 & 0.2 & 2.3 & 2.8 & 2.4 & 3.9 & 5.7 & 2.3 & 0.5 & 2.5 & 3.9 & 1.7 & 2.1 & 1.5 & 0.27 & 0.6 \\
\hline $\mathrm{S} 21$ & 7.0 & 0.4 & 2.9 & 2.7 & 1.5 & 4.0 & 6.2 & 2.4 & 0.5 & 2.9 & 4.7 & 2.7 & 1.9 & 1.6 & 0.33 & 0.7 \\
\hline $\mathrm{S} 22$ & 9.1 & 0.3 & 2.7 & 3.1 & 1.6 & 4.5 & 7.0 & 3.3 & 0.7 & 3.0 & 5.0 & 2.9 & 3.4 & 1.9 & 0.39 & 1.0 \\
\hline $\mathrm{S} 23$ & 8.5 & 1.3 & 4.6 & 1.6 & 2.5 & $\underline{1.8}$ & $\underline{3.5}$ & 3.5 & $\underline{1.4}$ & $\underline{1.3}$ & 2.3 & 3.0 & 3.6 & 0.7 & 0.10 & 1.4 \\
\hline $\mathrm{S} 24$ & 10.3 & 0.5 & 3.0 & 2.2 & $\underline{3.6}$ & 3.4 & 4.3 & 2.0 & 0.6 & 2.6 & 3.7 & 1.9 & 2.7 & $\underline{2.2}$ & 0.35 & 1.0 \\
\hline
\end{tabular}

The font colors of the sample ID cells correspond to the six clusters identified by the cluster analysis on the OM molecular composition dataset.

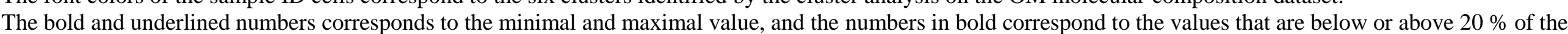
maximal and minimal values respectively. 
Table S4. Continuation Sediment OM molecular composition variables for the 42 sediment samples analysed by Py-GC/MS

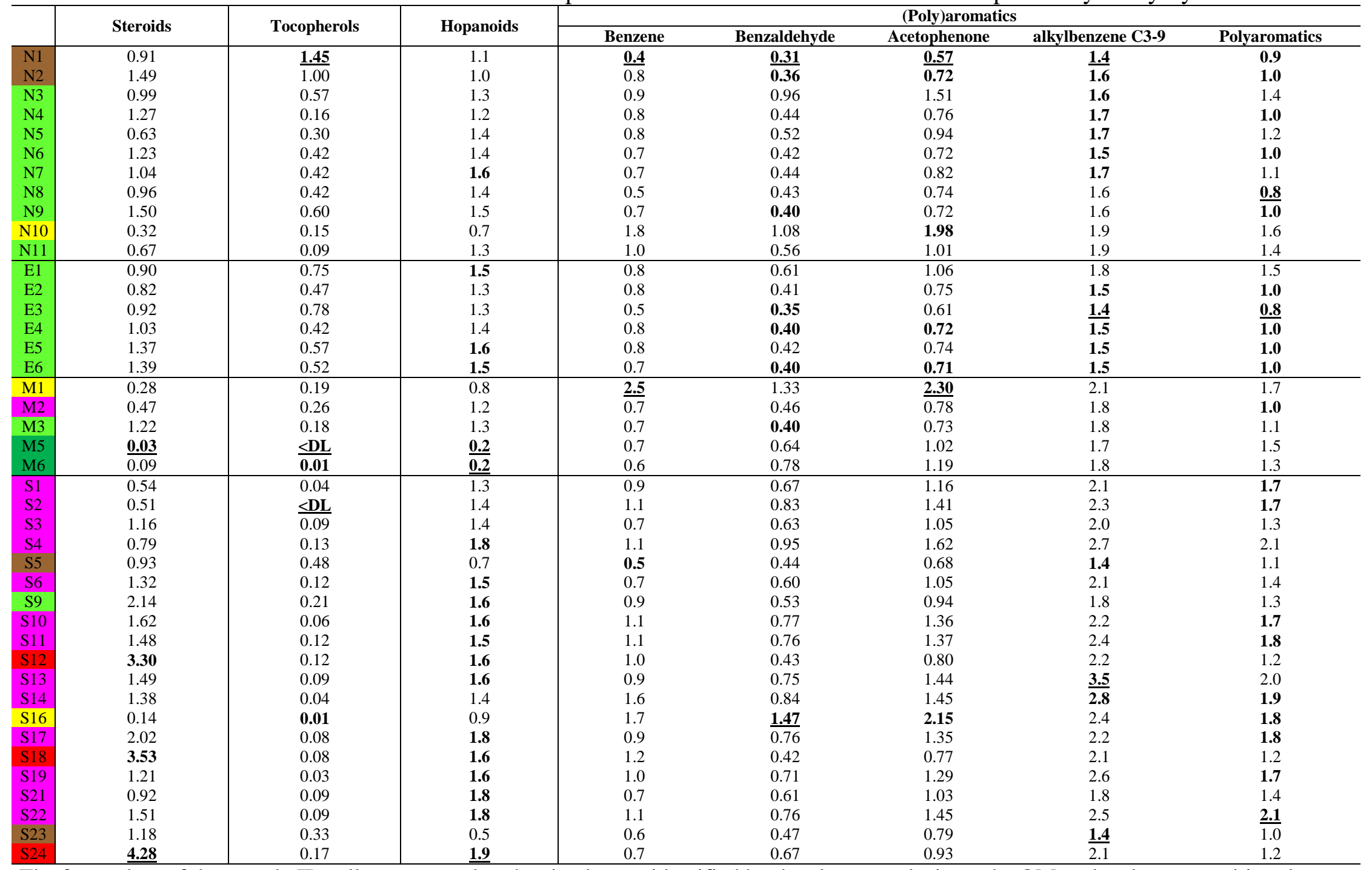

The font colors of the sample ID cells correspond to the six clusters identified by the cluster analysis on the OM molecular composition dataset. The bold and underlined numbers corresponds to the minimal and maximal value, and the numbers in bold correspond to the values that are below or above $20 \%$ of the maximal and minimal values respectively. 
Table S5. Average of the OM molecular composition variables for the whole-lake and the six clusters

\begin{tabular}{|c|c|c|c|c|c|c|c|}
\hline \multirow[b]{2}{*}{ Variables (unit) } & \multirow[b]{2}{*}{$\begin{array}{c}\text { Whole-lake }^{\mathrm{a}} \\
\left(\mathbf{n}^{\mathrm{b}}=\mathbf{4 2}\right)\end{array}$} & \multirow{2}{*}{$\begin{array}{c}\begin{array}{c}\text { Near-shore } \\
\text { sites }\end{array} \\
\text { Cluster }_{\mathrm{OM}} 5 \\
(\mathrm{n}=4)\end{array}$} & \multirow{2}{*}{$\begin{array}{c}\begin{array}{c}\text { North/East } \\
\text { basins }\end{array} \\
\text { Cluster }_{\mathrm{OM}} 1 \\
(\mathrm{n}=16)\end{array}$} & \multicolumn{2}{|c|}{ South basins } & \multicolumn{2}{|c|}{ Shallow central areas } \\
\hline & & & & $\begin{array}{c}\text { Cluster }{ }_{\mathrm{OM}} 3 \\
(\mathrm{n}=14)\end{array}$ & $\begin{array}{c}\text { Cluster }_{\mathrm{OM}} 2 \\
(\mathrm{n}=3)\end{array}$ & $\begin{array}{c}\text { Cluster }_{\mathrm{OM}} 4 \\
(\mathrm{n}=3)\end{array}$ & $\begin{array}{c}\text { Cluster }_{\mathrm{OM}} 6 \\
(\mathrm{n}=2)\end{array}$ \\
\hline \multicolumn{8}{|l|}{ Carbohydrates } \\
\hline (Alkyl)-furans \& furanones & $15 \pm 5$ & $12 \pm 4$ & $12 \pm 2$ & $17 \pm 3$ & $11.9 \pm 0.7$ & $22 \pm 5$ & $23 \pm 2$ \\
\hline $\begin{array}{l}\text { Hydroxy- or carboxy- } \\
\text { furans \& furanones }\end{array}$ & $4 \pm 1$ & $5.4 \pm 0.4$ & $4.2 \pm 0.7$ & $3.7 \pm 0.5$ & $3.7 \pm 0.1$ & $6.1 \pm 1.9$ & $1.2 \pm 0.6$ \\
\hline Pyrans & $3 \pm 1$ & $4.7 \pm 0.7$ & $3.6 \pm 0.9$ & $3 \pm 1$ & $3.0 \pm 0.3$ & $3.3 \pm 0.8$ & $1.4 \pm 0.4$ \\
\hline Dianhydrorhamnose & $1.6 \pm 0.5$ & $1.7 \pm 0.3$ & $1.7 \pm 0.4$ & $1.7 \pm 0.5$ & $1.6 \pm 0.2$ & $1.7 \pm 0.3$ & $0.5 \pm 0.2$ \\
\hline Levoglucosenone & $2.2 \pm 0.4$ & $2.3 \pm 0.4$ & $2.2 \pm 0.4$ & $2.2 \pm 0.4$ & $1.9 \pm 0.5$ & $2.8 \pm 0.3$ & $1.9 \pm 0.5$ \\
\hline Anhydrosugars & $4 \pm 3$ & $6.4 \pm 3.9$ & $4.9 \pm 2.3$ & $2.4 \pm 1.9$ & $2.2 \pm 0.6$ & $2.19 \pm 0.03$ & $1.02 \pm 0.06$ \\
\hline \multicolumn{8}{|l|}{ Chitin-derived compounds } \\
\hline Chitin-derived compounds & $3 \pm 1$ & $2.3 \pm 0.9$ & $2.7 \pm 0.8$ & $2.6 \pm 0.8$ & $3.8 \pm 0.4$ & $1.6 \pm 0.8$ & $0.23 \pm 0.1$ \\
\hline \multicolumn{8}{|l|}{ N-compounds } \\
\hline (alkyl)pyridines & $0.3 \pm 0.1$ & $0.37 \pm 0.03$ & $0.28 \pm 0.08$ & $0.35 \pm 0.06$ & $0.41 \pm 0.04$ & $0.16 \pm 0.07$ & $0.06 \pm 0.01$ \\
\hline Pyridines_O & $0.7 \pm 0.1$ & $0.63 \pm 0.06$ & $0.71 \pm 0.08$ & $0.79 \pm 0.06$ & $0.77 \pm 0.09$ & $0.7 \pm 0.2$ & $0.3 \pm 0.2$ \\
\hline (alkyl)pyrroles & $2.4 \pm 0.5$ & $1.9 \pm 0.1$ & $2.0 \pm 0.3$ & $2.8 \pm 0.4$ & $2.9 \pm 0.2$ & $2.8 \pm 0.6$ & $2.4 \pm 0.4$ \\
\hline Pyrroles_O & $1.0 \pm 0.2$ & $0.63 \pm 0.06$ & $0.9 \pm 0.1$ & $1.2 \pm 0.1$ & $0.90 \pm 0.04$ & $1.24 \pm 0.08$ & $0.6 \pm 0.2$ \\
\hline $\begin{array}{l}\text { Pyrroledione/ } \\
\text { pyrrolidinedione }\end{array}$ & $1.2 \pm 0.3$ & $1.2 \pm 0.08$ & $1.2 \pm 0.2$ & $1.1 \pm 0.3$ & $1.5 \pm 0.2$ & $1.5 \pm 0.2$ & $0.2 \pm 0.1$ \\
\hline Aromatic N & $0.8 \pm 0.3$ & $0.5 \pm 0.1$ & $0.6 \pm 0.1$ & $1.0 \pm 0.2$ & $1.0 \pm 0.4$ & $1.0 \pm 0.3$ & $0.9 \pm 0.3$ \\
\hline Indoles & $1.5 \pm 0.4$ & $1.39 \pm 0.08$ & $1.4 \pm 0.2$ & $1.7 \pm 0.1$ & $2.3 \pm 0.7$ & $1.5 \pm 0.2$ & $0.7 \pm 0.3$ \\
\hline Diketodipyrrole & $0.8 \pm 0.2$ & $1.0 \pm 0.2$ & $0.7 \pm 0.1$ & $0.78 \pm 0.08$ & $0.92 \pm 0.07$ & $0.5 \pm 0.2$ & $0.7 \pm 0.4$ \\
\hline Proteins & $1.5 \pm 0.4$ & $1.6 \pm 0.3$ & $1.6 \pm 0.2$ & $1.3 \pm 0.3$ & $2.5 \pm 0.2$ & $1.2 \pm 0.4$ & $0.6 \pm 0.5$ \\
\hline Alkylamides & $0.6 \pm 0.3$ & $0.46 \pm 0.09$ & $0.7 \pm 0.1$ & $0.5 \pm 0.2$ & $1.3 \pm 0.3$ & $0.3 \pm 0.2$ & $0.11 \pm 0.06$ \\
\hline \multicolumn{8}{|l|}{ Phenols and Lignins } \\
\hline Phenols & $8.2 \pm 1.2$ & $10 \pm 1$ & $7.7 \pm 0.7$ & $8.2 \pm 0.8$ & $9.2 \pm 0.9$ & $8 \pm 1$ & $6 \pm 2$ \\
\hline Guaiacols & $4 \pm 2$ & $9 \pm 4$ & $4 \pm 1$ & $2.7 \pm 0.5$ & $3.1 \pm 0.7$ & $1.9 \pm 0.1$ & $1.3 \pm 0.3$ \\
\hline Syringols & $0.5 \pm 0.4$ & $1.6 \pm 0.3$ & $0.5 \pm 0.3$ & $0.3 \pm 0.1$ & $0.7 \pm 0.4$ & $0.21 \pm 0.01$ & $0.1 \pm 0.1$ \\
\hline \multicolumn{8}{|l|}{ Chlorophylls } \\
\hline Pristenes & $2.7 \pm 0.8$ & $2.6 \pm 0.9$ & $3.1 \pm 0.6$ & $2.8 \pm 0.3$ & $2.4 \pm 0.2$ & $1.9 \pm 0.3$ & $0.5 \pm 0.1$ \\
\hline Phytadienes & $1.9 \pm 0.6$ & $1.7 \pm 0.6$ & $1.8 \pm 0.5$ & $1.9 \pm 0.5$ & $3.1 \pm 0.4$ & $1.3 \pm 0.2$ & $0.6 \pm 0.6$ \\
\hline \multicolumn{8}{|l|}{$n$-alkenes (Cn:1) } \\
\hline C9-16:1 & $3.5 \pm 0.8$ & $2.1 \pm 0.2$ & $3.2 \pm 0.4$ & $4.2 \pm 0.6$ & $3.3 \pm 0.2$ & $3.7 \pm 0.7$ & $3.9 \pm 0.3$ \\
\hline C17-C22:1 & $6 \pm 1$ & $5 \pm 1$ & $6.6 \pm 0.8$ & $6.4 \pm 0.5$ & $5.1 \pm 0.7$ & $5.7 \pm 0.5$ & $3.9 \pm 0.2$ \\
\hline C23-26_1 & $2.9 \pm 0.9$ & $3.3 \pm 0.6$ & $3.6 \pm 0.8$ & $2.5 \pm 0.4$ & $2.4 \pm 0.4$ & $2.2 \pm 0.7$ & $1.0 \pm 0.6$ \\
\hline $\mathrm{C} 27-28: 1$ & $0.8 \pm 0.4$ & $1.1 \pm 0.3$ & $1.1 \pm 0.2$ & $0.6 \pm 0.1$ & $0.9 \pm 0.2$ & $0.3 \pm 0.2$ & $0.12 \pm 0.02$ \\
\hline \multicolumn{8}{|l|}{$n$-alkanes (Cn:0) } \\
\hline C13-16:0 & $2.5 \pm 0.6$ & $1.7 \pm 0.3$ & $2.3 \pm 0.3$ & $3.0 \pm 0.5$ & $2.6 \pm 0.1$ & $2.7 \pm 0.3$ & $2.4 \pm 0.1$ \\
\hline $\mathrm{C} 17-22: 0$ & $3.9 \pm 0.8$ & $2.8 \pm 0.4$ & $4.0 \pm 0.5$ & $4.4 \pm 0.6$ & $3.9 \pm 0.2$ & $3.3 \pm 0.2$ & $1.9 \pm 0.4$ \\
\hline C23-26:0 & $3 \pm 1$ & $2.4 \pm 0.9$ & $3.2 \pm 0.8$ & $2.1 \pm 0.5$ & $2.1 \pm 0.1$ & $2.4 \pm 0.6$ & $8 \pm 1$ \\
\hline $\mathrm{C} 27-35: 0$ & $4 \pm 4$ & $3 \pm 2$ & $5 \pm 1$ & $2.3 \pm 0.8$ & $3.2 \pm 0.4$ & $2.8 \pm 1.4$ & $17 \pm 7$ \\
\hline \multicolumn{8}{|l|}{ Alkan-2-ones (2K) } \\
\hline $2 \mathrm{~K} \mathrm{C} 13-17$ & $1.3 \pm 0.4$ & $0.7 \pm 0.1$ & $1.1 \pm 0.2$ & $1.8 \pm 0.2$ & $1.7 \pm 0.4$ & $1.1 \pm 0.3$ & $1.60 \pm 0.02$ \\
\hline $2 \mathrm{~K} \mathrm{C} 19-22$ & $0.3 \pm 0.1$ & $0.18 \pm 0.06$ & $0.33 \pm 0.06$ & $0.4 \pm 0.1$ & $0.33 \pm 0.03$ & $0.13 \pm 0.07$ & $0.03 \pm 0.01$ \\
\hline $2 \mathrm{~K} \mathrm{C} 23-31$ & $1.3 \pm 0.8$ & $1.3 \pm 0.4$ & $2.1 \pm 0.7$ & $0.8 \pm 0.3$ & $1.1 \pm 0.1$ & $0.5 \pm 0.4$ & $0.2 \pm 0.2$ \\
\hline \multicolumn{8}{|l|}{ Steroids } \\
\hline Steroids & $1.2 \pm 0.9$ & $1.1 \pm 0.3$ & $1.1 \pm 0.4$ & $1.2 \pm 0.5$ & $3.7 \pm 0.5$ & $0.25 \pm 0.09$ & $0.06 \pm 0.04$ \\
\hline \multicolumn{8}{|l|}{ Tocopherols } \\
\hline Tocopherols & $0.3 \pm 0.3$ & $0.8 \pm 0.5$ & $0.4 \pm 0.2$ & $0.09 \pm 0.06$ & $0.12 \pm 0.04$ & $0.11 \pm 0.09$ & $0.01 \pm 0.01$ \\
\hline \multicolumn{8}{|l|}{ Hopanoids } \\
\hline Hopanoids & $1.3 \pm 0.4$ & $0.8 \pm 0.3$ & $1.4 \pm 0.1$ & $1.6 \pm 0.2$ & $1.7 \pm 0.2$ & $0.78 \pm 0.09$ & $0.17 \pm 0.01$ \\
\hline \multicolumn{8}{|l|}{ (Poly)aromatics } \\
\hline Benzene & $0.9 \pm 0.4$ & $0.6 \pm 0.2$ & $0.8 \pm 0.1$ & $1.0 \pm 0.3$ & $1.0 \pm 0.3$ & $2.0 \pm 0.4$ & $0.67 \pm 0.06$ \\
\hline Benzaldehyde & $0.6 \pm 0.3$ & $0.39 \pm 0.07$ & $0.5 \pm 0.1$ & $0.7 \pm 0.1$ & $0.5 \pm 0.1$ & $1.3 \pm 0.2$ & $0.71 \pm 0.09$ \\
\hline Acetylbenzene & $1.1 \pm 0.4$ & $0.7 \pm 0.1$ & $0.8 \pm 0.2$ & $1.3 \pm 0.2$ & $0.84 \pm 0.09$ & $2.2 \pm 0.2$ & $1.1 \pm 0.1$ \\
\hline Alkylbenzenes C3-9 & $1.9 \pm 0.5$ & $1.44 \pm 0.09$ & $1.6 \pm 0.2$ & $2.4 \pm 0.4$ & $2.10 \pm 0.04$ & $2.1 \pm 0.2$ & $1.71 \pm 0.07$ \\
\hline Polyaromatics & $1.4 \pm 0.4$ & $1.01 \pm 0.07$ & $1.1 \pm 0.2$ & $1.7 \pm 0.3$ & $1.21 \pm 0.03$ & $1.7 \pm 0.1$ & $1.4 \pm 0.1$ \\
\hline
\end{tabular}

a whole-lake: averages of all analyzed sediment samples excluding the two outlier samples (sites M4, S15); ${ }^{b}$ : number of sample; The six clusters are presented in Fig. 1d in the manuscript.

Light grey background denotes average values below $10 \%$ of whole-lake average. No background denotes values close to whole-lake average $( \pm 10 \%)$. Dark grey background denotes average values above $10 \%$ of whole-lake average. 